\title{
EARLY-TYPE GALAXIES AT $z=1.3$. I. THE LYNX SUPERCLUSTER: CLUSTER AND GROUPS AT $z=1.3$. MORPHOLOGY AND COLOR-MAGNITUDE RELATION
}

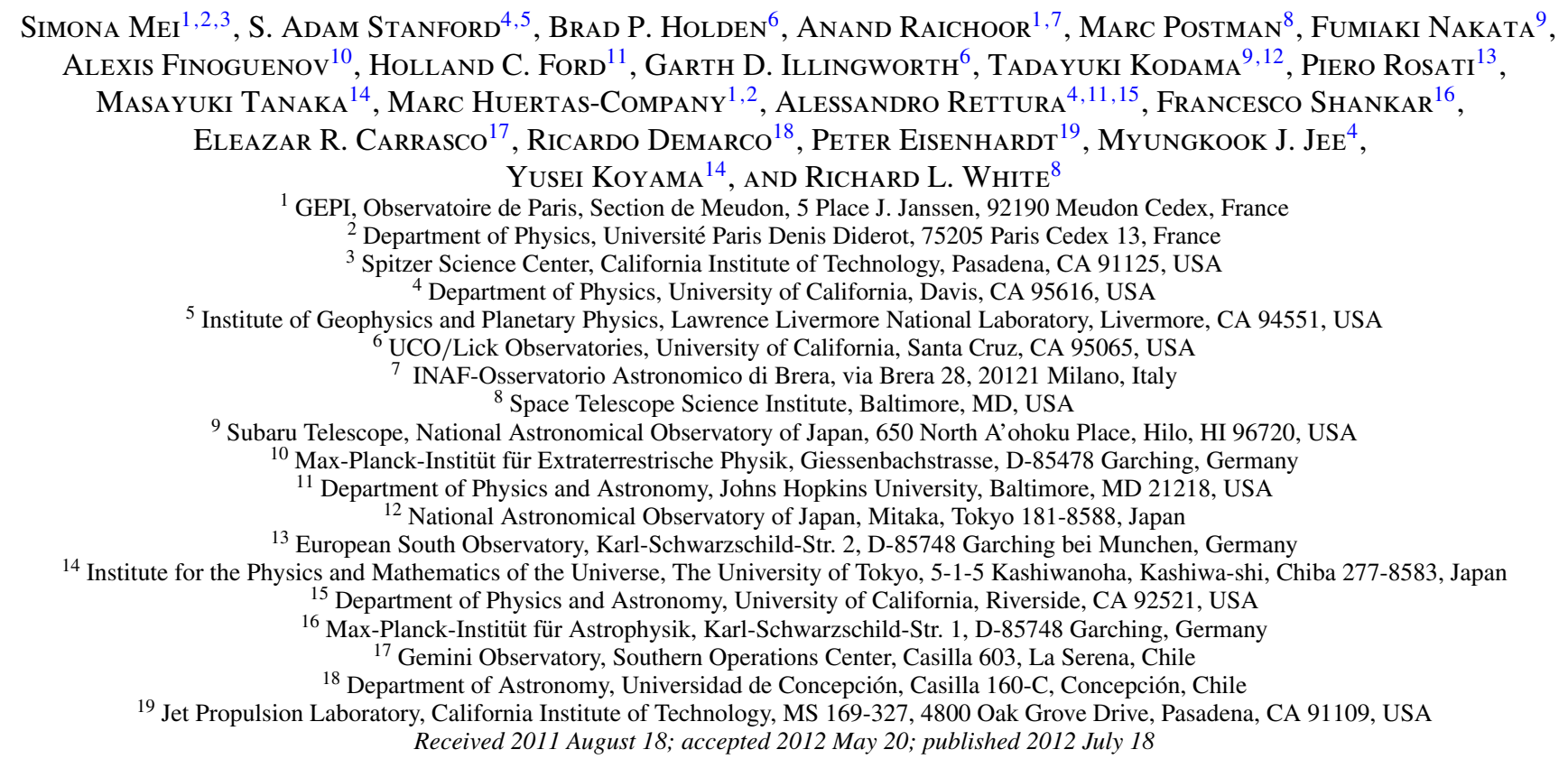

\begin{abstract}
We confirm the detection of three groups in the Lynx supercluster, at $z \approx 1.3$, through spectroscopic follow-up and $\mathrm{X}$-ray imaging, and we give estimates for their redshifts and masses. We study the properties of the group galaxies compared to the two central clusters, RX J0849+4452 and RX J0848+4453. Using spectroscopic follow-up and multi-wavelength photometric redshifts, we select 89 galaxies in the clusters, of which 41 are spectroscopically confirmed, and 74 galaxies in the groups, of which 25 are spectroscopically confirmed. We morphologically classify galaxies by visual inspection, noting that our early-type galaxy (ETG) sample would have been contaminated at the 30\%-40\% level by simple automated classification methods (e.g., based on Sérsic index). In luminosity-selected samples, both clusters and groups show high fractions of bulge-dominated galaxies with a diffuse component that we visually identified as a disk and which we classified as bulge-dominated spirals, e.g., Sas. The ETG fractions never rise above $\approx 50 \%$ in the clusters, which is low compared to the fractions observed in other massive clusters at $z \approx 1$. In the groups, ETG fractions never exceed $\approx 25 \%$. However, overall bulge-dominated galaxy fractions (ETG plus Sas) are similar to those observed for ETGs in clusters at $z \sim 1$. Bulge-dominated galaxies visually classified as spirals might also be ETGs with tidal features or merger remnants. They are mainly red and passive, and span a large range in luminosity. Their star formation seems to have been quenched before experiencing a morphological transformation. Because their fraction is smaller at lower redshifts, they might be the spiral population that evolves into ETGs. For mass-selected samples of galaxies with masses $M>10^{10.6} M_{\odot}$ within $\Sigma>500 \mathrm{Mpc}^{-2}$, the ETG and overall bulge-dominated galaxy fractions show no significant evolution with respect to local clusters, suggesting that morphological transformations might occur at lower masses and densities. The ETG mass-size relation shows evolution toward smaller sizes at higher redshift in both clusters and groups, while the late-type mass-size relation matches that observed locally. When compared to the clusters, the group ETG red sequence shows lower zero points (at $\sim 2 \sigma$ ) and larger scatters, both expected to be an indication of a younger galaxy population. However, we show that any allowed difference between the age in groups and clusters would be small when compared to the differences in age in galaxies of different masses.
\end{abstract}

Key words: galaxies: clusters: general - galaxies: clusters: individual (the Lynx cluster) - galaxies: evolution galaxies: high-redshift - galaxies: stellar content - galaxies: structure

Online-only material: color figures

\section{INTRODUCTION}

The currently favored cosmological model, $\Lambda \mathrm{CDM}$ (e.g., Komatsu et al. 2011), predicts that some of the galaxies in clusters formed at the peaks of dark matter fluctuations (a pristine population) while others were accreted from surrounding infalling groups and the field (infalling population). During this accretion process, we expect galaxy interactions with the environment to quench star formation and trigger a stellar population (from active to passive) and morphological (from late- to early-type) transformation (e.g., Diaferio et al. 2001; De Lucia et al. 2006; Poggianti et al. 2006, 2010; Faber et al. 
2007; Moran et al. 2007). Various transformation mechanisms have been proposed, some driven by the galaxy environmentincluding merging, galaxy harassment, and gas stripping-some related to intrinsic galaxy properties, such as mass, and still others related to both the galaxy environment and intrinsic galaxy properties (for a review see Boselli \& Gavazzi 2006; for a critical summary of transformation mechanisms in clusters, see Treu et al. 2003).

The primary mechanisms driving galaxy transformations in clusters arise from galaxy-galaxy and galaxy-cluster (mainly tidal) interactions, dynamical friction, and interaction with the hot cluster medium (ram pressure stripping, strangulation). Dynamical friction drags galaxies toward the cluster center where they can merge with the central galaxy. For galaxy-galaxy mergers to occur, however, the internal velocity dispersion of the galaxies must be greater than their orbital energy. In galaxy clusters, where cluster velocity dispersions are high, galaxy mergers are therefore unlikely, while groups present an ideal environment for such interactions.

$\Lambda C D M$ semianalytical models (e.g., McGee et al. 2009) predict that $\approx 40 \%$ of galaxies in a Coma-sized cluster at $z=0.5$ are accreted from groups with masses larger than $M=10^{13} M_{\odot}$. At higher redshift, the number of galaxies accreted by a cluster and the fraction of galaxies that suffered a transformation by the environment are both lower. This model implies that at $z \approx 1.5$, environmental effects should not yet be significant, but will be predominant only at $z<1.5$.

Moreover, both star formation and morphological transformations are observed to occur mainly at redshifts $z<1$ when star formation decreases and the early-type population increases. While the elliptical fractions show little evolution, this increase is due to a lack of S0 galaxies at higher redshift (e.g., Postman et al. 2005; Poggianti et al. 2006, 2010; Moran et al. 2007).

Both models and observations agree that at high redshift, e.g., $z>1$, we should observe galaxies before or during these transformations and structures in the very first phases of their assembly. As a result, we can observe galaxies in groups before their infall into the denser cluster environment and disentangle galaxy transformations occurring in different environments.

Recent work (e.g., Poggianti et al. 2009; Just et al. 2010) has shown that the evolution of the S0 galaxy fraction is stronger in poor clusters and groups compared to more massive clusters over the redshift range $0<z<0.5$, suggesting that transformation mechanisms associated with these environments (e.g., galaxy-galaxy interactions as opposed to galaxy-cluster interactions) dominate the S0 transformations.

At higher redshifts $(0.4<z<1.2)$, however, Capak et al. (2007) have shown that early-type galaxy fractions change faster in high-density environments. Since this is the range in redshift where most of the group accretion takes place in $\Lambda \mathrm{CDM}$, we would like to understand which galaxies are accreted by galaxy clusters at this epoch. Their morphology and stellar population before accretion should provide clues to understanding how the accretion process changes the high-density population. With this aim, we have studied in detail the cluster and group population of a supercluster at $z \approx 1.3$, the Lynx supercluster.

Superclusters are the largest structures observed in the universe and the ideal laboratory for studying galaxy transformations in different environments. Their dimensions can range from 10 to $\approx 100 \mathrm{Mpc}$, and they are composed of two or more galaxy clusters and surrounding groups. Studies of these large structures at high redshift have proliferated in recent years (e.g., Gal et al. 2008; Lubin et al. 2009; Tanaka et al. 2009) and re- veal the different star formation rates and histories of galaxy populations in different environments at $z \approx 1$. Only in recent years have superclusters been discovered at redshifts $z>1$.

Thanks to recent wide-field multi-wavelength imaging with the Suprime-Cam on the Subaru telescope, and infrared followup, the first supercluster at $z \approx 1.3$ was discovered by Nakata et al. (2005), the Lynx supercluster, followed by the discovery of a supercluster around RDCS J1252.9-2927 $z=1.24$ by Tanaka et al. (2007). A recent paper by Tanaka et al. (2009) confirmed this last superstructure through spectroscopic follow-up with FORS2 and GMOS on Gemini-South, and presented detailed studies of the cluster and group galaxy star formation activity. Their results show a population of red star-forming galaxies in the groups that are not found in the central cluster. They interpret their observed spectral line strengths and colors by the presence of star formation heavily obscured by dust. They suggest that this is driven by galaxy-galaxy interactions that are more active in galaxy groups.

In this paper we study galaxy transformations in the Lynx supercluster at $z=1.26$, confirming the detection of a supercluster with spectroscopic follow-up and X-ray imaging.

The Lynx supercluster is composed of two clusters and numerous surrounding groups. The two clusters, RX J0849+4452 (the eastern cluster, hereafter Lynx E) and RX J0848+4453 (the western cluster, hereafter Lynx W), were detected in the near-infrared (Lynx W; Stanford et al. 1997) and through their X-ray emission in the ROSAT Deep Cluster Survey (Rosati et al. 1999), and spectroscopically confirmed respectively at $z=1.261$ (Rosati et al. 1999) and $z=1.273$ (Stanford et al. 1997).

While Lynx E presents a more compact galaxy distribution, with a central bright galaxy merger (van Dokkum et al. 2001; Yamada et al. 2002; that might eventually lead to a central $\mathrm{cD}$, cluster-dominant galaxy), the galaxies in Lynx $\mathrm{W}$ are more sparsely distributed in a filamentary structure and do not present an obvious central bright $\mathrm{cD}$ galaxy. Their X-ray emission as seen by Chandra (Stanford et al. 2001) follows the optical distribution, with Lynx E showing a more compact spherical shape and Lynx W a more elongated one, and luminosities of $L_{X}^{\text {bol }}=(2.8 \pm 0.2) \times 10^{44} \mathrm{erg} \mathrm{s}^{-1}$ and $L_{X}^{\text {bol }}=(1.0 \pm 0.7) \times$ $10^{44} \mathrm{erg} \mathrm{s}^{-1}$, respectively (Rosati et al. 1999; Stanford et al. 2001; Ettori et al. 2004). Together with a more compact galaxy distribution, this is an indication that Lynx $\mathrm{E}$ is likely to be more dynamically evolved with respect to Lynx W. The velocity dispersion for Lynx W was measured at $\sigma=650 \pm 170 \mathrm{~km} \mathrm{~s}^{-1}$ (Stanford et al. 2001). This value is consistent with estimates from the Jee et al. (2006) weak lensing analysis of $\sigma=$ $740_{-134}^{+113} \mathrm{~km} \mathrm{~s}^{-1}$ and $\sigma=762_{-133}^{+113} \mathrm{~km} \mathrm{~s}^{-1}$ for Lynx E and Lynx W, respectively. The most recent estimates of the cluster temperatures are $T=3.8_{-0.7}^{+1.3} \mathrm{keV}$ and $T=1.7_{-0.7}^{+1.3} \mathrm{keV}$, respectively (Jee et al. 2006 measurements that are consistent with Stanford et al. 2001).

Deep, panoramic multi-color ( $V R i^{\prime}, z^{\prime}$ bands) imaging with Suprime-Cam on the Subaru telescope identified seven galaxy groups around these two central clusters (Nakata et al. 2005). This makes the Lynx region a unique laboratory, being one of the very few superclusters observed at such a high redshift, and for this reason one of the best regions at $z>1$ in which we study the properties of evolving galaxies in different environments within a structure that is in the process of assembly.

This paper is the first of a series of papers centered on the Lynx supercluster, and will be followed by an analysis of galaxy masses and ages (Raichoor et al. 2011, Paper II), 
their star formation histories compared to the field (Rettura et al. 2011; hereafter Paper III), and the evolution of the Kormendy and mass-size relations in different environments (Raichoor et al. 2012, hereafter Paper IV). In this paper, we will mainly concentrate on galaxy morphology and the buildup of the color-magnitude relation (CMR). Our observations are presented in Section 2. In Section 3 we describe our sample selection. In Section 4 we describe our galaxy morphology and color measurements. In Section 5, we present and discuss our results, and we conclude with Section 6.

We adopt the Wilkinson Microwave Anisotropy Probe $(W M A P)$ cosmology $\left(\Omega_{m} h^{2}=0.1334, \Omega_{\Lambda}=0.734, h=0.71\right.$; Komatsu et al. 2011). All Advanced Camera for Surveys (ACS) filter magnitudes are given in the AB system (Oke \& Gunn 1983; Sirianni et al. 2005).

\section{OBSERVATIONS}

\subsection{Imaging and Spectroscopy}

We have observed the main two clusters of the Lynx superstructure as part of the ACS Intermediate Redshift Cluster Survey (IRCS; Postman et al. 2005), in the F775W (from hereafter $i_{775}$ ) and F850LP (from here after $z_{850}$ ) bandpasses with the ACS Wide Field Camera (WFC) in 2004 March and April, for a total of 7300 and 12,200 s, respectively. The surrounding Lynx groups were observed with the ACS WFC as part of an HST GO program (PI: Mei) between 2005 December and 2006 February, in the same bandpasses, $i_{775}$ and $z_{850}$, for a total of 6300 and 10,500 s, respectively. The ACS WFC resolution (pixel size) is $0{ }^{\prime} .05$ pixel $^{-1}$ and its field of view is $202^{\prime \prime} \times$ $202^{\prime \prime}$. The ACS images were processed with the APSIS pipeline (Blakeslee et al. 2003a, 2003b), with a Lanczos3 interpolation kernel. Our photometry was calibrated to the AB system, with synthetic photometric zero points of 25.678 and $24.867 \mathrm{mag}$, respectively, in $i_{775}$ and $z_{850}$ from the $H S T /$ ACS Web site. ${ }^{20} \mathrm{~A}$ reddening of $E(B-V)=0.027$ was adopted (Schlegel et al. 1998), with $A_{i 775}=0.054$ and $A_{z 850}=0.040$.

Spitzer/IRAC (Fazio et al. 1998) [3.6 $\mu \mathrm{m}$ ] and [4.5 $\mu \mathrm{m}$ ] band imaging of the clusters was obtained in 2004 April in 30 exposures of $200 \mathrm{~s}$ each for a total exposure time of $6000 \mathrm{~s}$. Lynx groups were observed in 2005 November and 2006 May, in 12 exposures of $100 \mathrm{~s}$ each, for a total exposure time of $1200 \mathrm{~s}$ (PI: S. A. Stanford). The data were reduced using standard Spitzer procedures. The basic calibrated data (hereafter BCD) frames were first corrected for muxbleed and pull down using custom IDL scripts (now available from the Spitzer Science Center), and then processed with MOPEX to produce co-added mosaics.

Spitzer/MIPS $24 \mu \mathrm{m}$ were also used, from program 83 (PI: Rieke; AORs 4758016 and 4758784), and were observed on 2004 April 9 in the photometry mode. To produce a mosaic, we combined the pointings toward the two clusters in the larger Lynx supercluster. The BCD frames were combined using the available MOPEX software from the Spitzer Science Center in 2006. The processing required eliminating the first two BCD frames in each exposure sequence and constructing an effective flatfield frame to remove some detector artifacts. A total of 224 BCD frames were combined into a single mosaic with 2". 45 pixels. We used the available mask files to eliminate bad pixels. The final mosaic had median background value of $35.21 \mathrm{MJy} \mathrm{sr}^{-1}$ with a dispersion of $0.018 \mathrm{MJy} \mathrm{sr}^{-1}$ or $2.53 \mu \mathrm{Jy} \mathrm{pixel}^{-1}$. The mosaic was aligned with the existing ACS imaging using sources in common between the two mosaics.
Using the software APEX, we constructed a source catalog of the mosaic. The resulting catalog has a $5 \sigma$ flux limit of $68 \mu \mathrm{Jy}$ at the deepest part of the mosaic. However, because of source confusion and the low effective exposure times in the rest of the data, the effective flux limit of the catalog is $125 \mu \mathrm{Jy}$.

The entire supercluster has been observed with SuprimeCam at the Subaru telescope in the $V, R, i^{\prime}$, and $z^{\prime}$ bandpasses between 2000 November and 2001 March. The Suprime-Cam has a field of view of $27 \times 27 \mathrm{arcmin}^{2}$ with a resolution of 0.2 arcsec pixel $^{-1}$. Details can be found in Nakata et al. (2005). These observations have been used to obtain galaxy photometric redshifts and to identify seven groups around the two main clusters. We will refer to Groups 1 through 7, with the same identifications as given in Nakata et al. (2005).

To complement space and Suprime-Cam observations, we added to our analysis ground-based observations in the $R, J$, and $K s$ bands (PI: S. A. Stanford), described in our companion paper, Paper II. Here, we briefly summarize these observations. The two clusters were observed in the $R$ band with the red camera on the Keck/LRIS Spectrograph (Oke et al. 1995), for a total exposure time of 8750 s. The groups have Palomar/ COSMIC (Kells et al. 1998) $R$-band imaging, obtained in 1999 November with the COSMIC instrument, for a total exposure time of $16,200 \mathrm{~s}$. The near-infrared $J-$ and $K_{s}$-band imaging was obtained in 2003 December at the KPNO $2.1 \mathrm{~m}$ telescope with the FLAMINGOS instrument (Elston 1998), for a total exposure time of about $24,000 \mathrm{~s}$ and $26,700 \mathrm{~s}$, respectively.

The imaging observations were followed by spectroscopic campaigns at the Subaru, Keck, and Gemini telescopes.

Cluster spectroscopy was obtained from published data (nine members from Stanford et al. 1997 and eight from Rosati et al. 1999) and spectroscopic runs at the Keck telescope with the Low Resolution Imaging Spectrograph (LRIS; Oke et al. 1995) and the Deep Imaging Multiobject Spectrograph (DEIMOS; for details see Mei et al. 2006b). In total, we have 41 confirmed cluster members with spectroscopic redshifts between 1.25 and 1.28.

Group spectroscopy was obtained from the Subaru, Keck, and Gemini telescopes. F.N. conducted a spectroscopic followup observation on 2007 January with FOCAS (Kashikawa et al. 2002) in MOS mode, at the Subaru telescope. We used a 300 line $\mathrm{mm}^{-1}$ grating blazed at $6500 \AA$ with the order-cut filter SO58. The wavelength coverage was between $5800 \AA$ and $10000 \AA$ with a pixel resolution of $1.34 \AA$ pixel $^{-1}$. A slit width was set to 0 . 8 , which gave a resolution of $\lambda / \Delta \lambda \sim 500$. We selected four FOCAS fields which efficiently cover the large-scale structure found by Nakata et al. (2005). Target galaxies were primarily selected on the basis of their photometric redshifts, as in Nakata et al. Observing conditions were good with $\sim 0.6-0.9$ arcsec seeing. Total on-source exposures ranged between $8100 \mathrm{~s}$ and 14,400 s. Data reduction was performed by F.N. in a standard manner using IRAF. All the reduced one-dimensional/twodimensional spectra were visually inspected to estimate the redshift of each object. In this work, we only use spectroscopic redshifts with a good quality measurement.

Keck spectroscopy was performed with the instrument DEIMOS by A.S. and B.H. on UT 2009 October 18 and 19 to cover Groups 1 and 2. On October 18, three exposures of $1800 \mathrm{~s}$ each were obtained when conditions were clear with 0.7 arcsec seeing. On October 19, four exposures of $1800 \mathrm{~s}$ were obtained when conditions were clear with 0.9 arcsec seeing. The data were reduced by B.H. using the DEEP pipeline.

\footnotetext{
20 http://www.stsci.edu/hst/acs/
} 
Table 1

Lynx Supercluster

\begin{tabular}{|c|c|c|c|c|c|c|c|}
\hline Cluster/Group & $z$ & $\begin{array}{c}\sigma_{v}^{\mathrm{a}} \\
\left(\mathrm{km} \mathrm{s}^{-1}\right)\end{array}$ & $\begin{array}{c}L_{\mathrm{bol}}^{X \text { a }} \\
\left(10^{44} \mathrm{erg} \mathrm{s}^{-1}\right)\end{array}$ & $\begin{array}{l}R_{200} \mathrm{~b} \\
(\mathrm{Mpc})\end{array}$ & $\begin{array}{c}M_{\mathrm{tot}}^{X \mathrm{c}} \\
\left(10^{14} M_{\odot}\right)\end{array}$ & $N_{\text {zphot }}{ }^{\mathrm{d}}$ & $N_{\text {zspec }}{ }^{\mathrm{d}}$ \\
\hline RX J0849+4452 & 1.261 & $740_{-134}^{+113}$ & $2.83 \pm 0.17$ & 0.9 & $2.9 \pm 1.5$ & 49 & 19 \\
\hline RX J0848+4453 & 1.270 & $650 \pm 170$ & $1.04 \pm 0.73$ & 0.8 & $1.4 \pm 1.0$ & 40 & 22 \\
\hline Group 1 & 1.262 & & $<0.20 \pm 0.09$ & $<0.5$ & $<0.45 \pm 0.14$ & 31 & 9 \\
\hline Group 2 & 1.260 & & $0.28 \pm 0.22$ & 0.5 & $0.57 \pm 0.26$ & 15 & 7 \\
\hline Group 3 & 1.263 & & $0.23 \pm 0.13$ & 0.5 & $0.50 \pm 0.17$ & 28 & 9 \\
\hline
\end{tabular}

Notes.

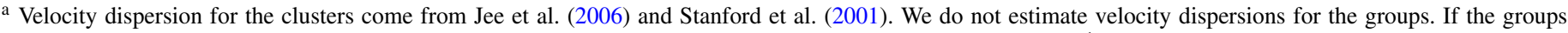

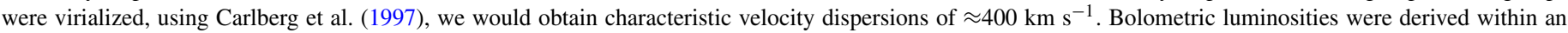
overdensity $\Delta_{z}=500$ for an Einstein-de Sitter universe from Ettori et al. (2004).

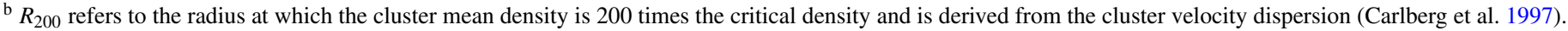

c Total masses were estimated out to $R_{500}$ using a cluster $\beta$ model together with the measured emission-weighted X-ray temperature (Ettori et al. 2004).

${ }^{\mathrm{d}} N_{\text {zphot }}$ and $N_{\text {zspec }}$ are the photometrically and spectroscopically selected members for each structure, respectively.

We obtained $20 \mathrm{hr}$ of spectroscopic observations with GMOS-N at the Gemini North Telescope, between 2006 January and April, on five (Group 1, Group 2, Group 3, Group 6, and Group 7) of the original seven groups observed for a total of $\approx 14,400 \mathrm{~s}$ each. These observations were reduced by S.M., R.C., and R.D. with the standard Gemini IRAF package released from the Gemini Observatory.

From the spectroscopic redshifts obtained at Subaru and Gemini, Groups 6 and 7 from Nakata et al. (2005) were confirmed to be structures at $z<1.26$. Three groups, Groups 1,2 , and 3 were confirmed to be at the same redshift as the two main clusters. The two clusters have a relative projected distance of $\approx 2 \mathrm{Mpc}$ and the two clusters and three spectroscopically confirmed groups (see below) cover a scale of around $30 \mathrm{Mpc}^{2}$, in the WMAP cosmology (Komatsu et al. 2011).

This paper will concentrate only on those three spectroscopically confirmed groups, whose properties are summarized in Table 1.

\subsection{XMM-Newton Imaging}

We retrieved XMM-Newton imaging (Jansen et al. 2001; ObsIDs 0085150101, 0085150201, and 0085150301) covering the Lynx supercluster region to evaluate group detection and estimate masses. We use the data collected by the European Photon Imaging Cameras (EPIC): the pn-CCD camera (Strüder et al. 2001) and the MOS-CCD cameras M1 and M2 (Turner et al. 2001), with total exposure time of $150 \mathrm{ks}$ before cleaning, and net time of $70.0 \mathrm{ks}, 89.8 \mathrm{ks}, 90.7 \mathrm{ks}$, respectively, after cleaning (see below).

Initial data processing was performed using the XMMSAS version 7.1 (Watson et al. 2001; Kirsch et al. 2004; Saxton et al. 2005). We then remove time intervals affected by solar flares to create the calibrated event file, as in Zhang et al. (2004), and apply the four-stage background subtraction of Finoguenov et al. (2007) to increase our capability to detect extended emission. High background in some of the MOS chips (Kuntz \& Snowden 2008) was checked and no instances were found. To create a final mosaic of cleaned images in the area, we estimate the background in each image for each instrument and correct for differences in sensitivity between instruments.

The residual map of extended sources is obtained by subtracting a point-spread function (PSF) model using the detected point sources from the background-subtracted image and smoothing the image with a Gaussian kernel of $32^{\prime \prime}$ width. The same tech- nique has been used to estimate the emission in Tanaka et al. (2010) and Gobat et al. (2011).

Figure 1 shows the $X M M$ composite image. We detect Group 3 at $2.6 \sigma$, Group 2 at $1.6 \sigma$. Group 1 shows a $0.5 \sigma$ excess, and there is an excess of the X-ray emission (at around $2 \sigma$ ) just close to it. From the X-ray emission and scaling relations (Markevitch et al. 1998; see also Bielby et al. 2010 for a detailed description of our X-ray analysis), we obtain a mass estimate for Groups 2 and 3 of $(5.7 \pm 2.6) \times 10^{13} M_{\odot}$ and $(5.0 \pm 1.7) \times 10^{13} M_{\odot}$, respectively, and a $95 \%$ upper limit for the mass of Group 1 of $M<4.5 \times 10^{13} M_{\odot}$.

\section{CLUSTER AND GROUP SAMPLE SELECTION}

Our ACS images were aligned on the center of the cluster X-ray emission from Stanford et al. (2001), for the two clusters. For the groups, our ACS images were aligned on the center of the density overdensities as estimated from Nakata et al. from a 10 nearest neighbor algorithm. For this analysis, we selected cluster and group galaxies using newly estimated photometric redshifts from our space- and ground-based data, and a Friend-of-Friends algorithm (FoF; Geller \& Huchra 1983; see also Postman et al. 2005 ) with scale derived from the two clusters (see below).

As a first step in the galaxy selection, we consider as potential group members all galaxies with Subaru $V r i^{\prime} z^{\prime}$ photometric redshifts $0.8<z_{\text {phot }}<1.4$ from Nakata et al. (2005), ACS magnitude brighter than $z_{850}=24 \mathrm{mag}$ (the limit magnitude for dependable visual morphological classification; see Postman et al. 2005), and ACS color $0.4<\left(i_{775}-z_{850}\right)<1.4$. This first selection is larger than the initial $1<z_{\text {phot }}<1.35$ range proposed by Nakata et al. (2005). It is based on the range in Subaru photometric redshifts and ACS colors covered by spectroscopically confirmed early- and late-type cluster members from our spectroscopic follow-up (see below) and the large range of ACS colors predicted from Bruzual \& Charlot (2003, hereafter BC03) stellar population models for early and star-forming galaxies at $z=1.26$. With this choice we obtain 195 objects with estimated completeness at more than $90 \%$ (from the spectroscopic follow-up and the photometric redshift uncertainties) at our limiting magnitude $z_{850}=24 \mathrm{mag}$.

From our spectroscopic runs on the groups, we obtained 52 redshift measurements, of which 25 are spectroscopically confirmed members with redshifts between 1.25 and 1.28 , with average spectroscopic redshift $z=1.262 \pm 0.007$ (Group 1; from nine members), $z=1.260 \pm 0.006$ (Group 2; from seven members), and $z=1.263 \pm 0.005$ (Group 3; from nine 


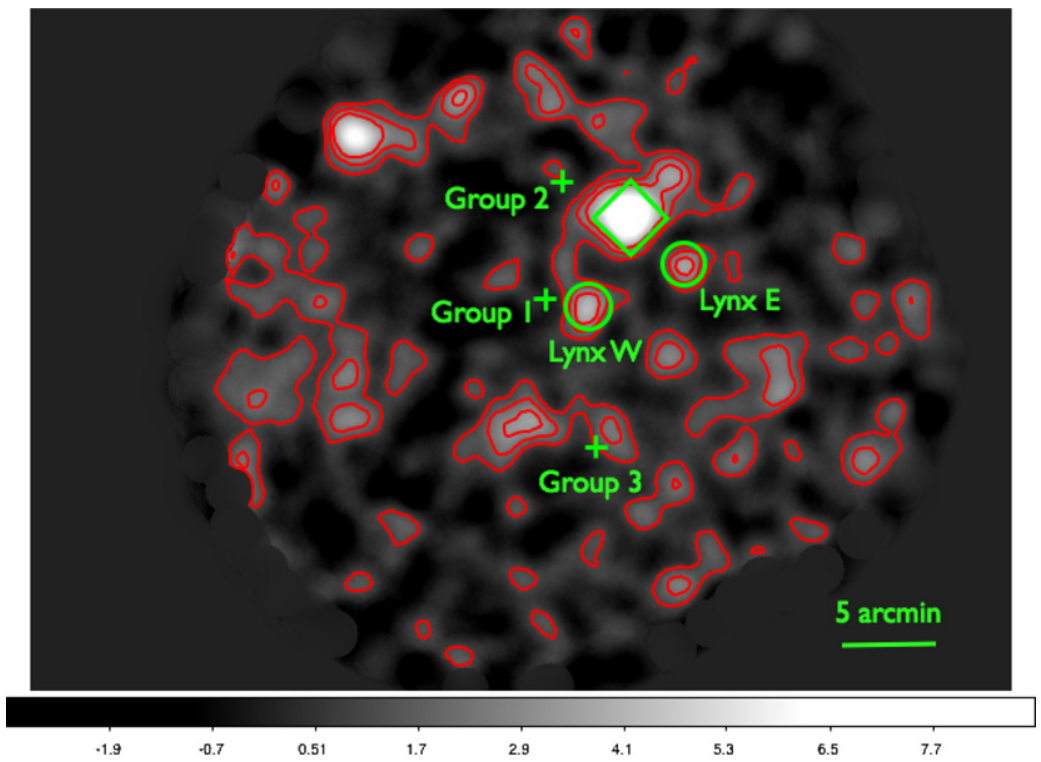

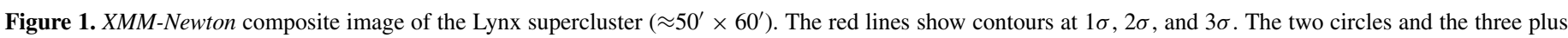

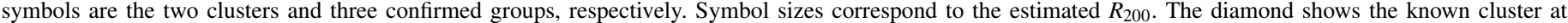

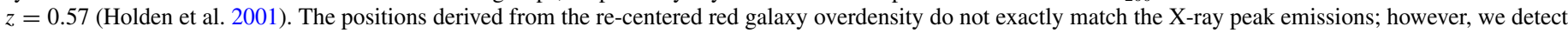

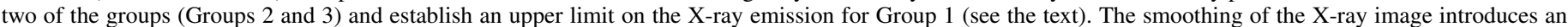
uncertainty in astrometry of $32^{\prime \prime}$.

(A color version of this figure is available in the online journal.)

members). These values are slightly different from those published in Paper II because we have obtained new spectroscopically confirmed members since publication.

Using our spectroscopic redshifts, we then eliminated from this sample all 27 spectroscopically confirmed outliers (9 in Group 1, 11 in Group 2, and 7 in Group 3). The contamination rate in all group spectroscopic samples, selected with Subaru photometric redshifts, is $\approx 50 \%$ and similar for the early- and late-type (see below for morphological classification) samples.

For the remaining 168 objects, we eliminated SExtractor (Bertin \& Arnouts 1996) multiple identifications (in the ACS images) and derived a new set of photometric redshifts using our space- and ground-based multi-wavelength photometry (in the range $0.6-4.5 \mu \mathrm{m}$ ) as described in our companion paper, Paper II. We also re-estimated photometric redshifts for the 94 cluster galaxies selected with the same criteria in color and photometric redshift.

To estimate new photometric redshifts, we used the photometry from Raichoor et al. (2011; see this paper for details on photometry and the sky background subtraction) and the public software Le Phare (Arnouts et al. 2002; Ilbert et al. 2006), based on a $\chi^{2}$ template fitting method. The best photometric redshift is calculated using the median of the probability distribution function. For our input parameters, we followed Ilbert et al. (2010): BC03 templates, solar metallicity, an exponentially decaying star formation with $\tau$ in the range 0.1-5 Gyr, and a Calzetti et al. (2000) extinction law with $E(B-V)$ in the range $0-0.5$.

These new photometric redshifts include optical photometry, ACS $i_{775}$ and $z_{850}$, near-IR and intermediate-IR photometry from Spitzer/IRAC, and help us to better define the statistics of our sample. In total we have 41 and 25 spectroscopically confirmed members in the clusters and groups, respectively, for a total of 66 spectroscopically confirmed members.

At $z=1.3$, photometric redshift uncertainties are larger than a galaxy group extension in redshift space (e.g., Evrard et al. 2008; George et al. 2011). We use an empirical approach to estimate the completeness and purity of our sample. Most $(90 \%$ of the entire sample; $95 \%$ of the early type, and $83 \%$ of the late type) of our spectroscopically confirmed members show new photometric redshifts in the range $0.92<z_{\text {phot }}<1.36$. The remaining $10 \%$ show $1.36<z_{\text {phot }}<2$. We use this new range in photometric redshift (e.g., $0.92<z_{\text {phot }}<1.36$ ) to select our final sample. In this range, we also find only 12 group outliers, making our estimated contamination level decrease from $50 \%$ to $\approx 20 \%$ with this new photometric redshift selection. From these empirical estimates, we consider our sample to be $\approx 95 \%$ (for the early-type galaxies) and $\approx 83 \%$ (for the late-type galaxies) complete and contaminated at $\approx 20 \%$.

To verify these estimates, we used as test sample the George et al. (2011) COSMOS group and field sample that uses Ilbert et al. (2009) photometric redshifts obtained with SED fitting using 30 bandpasses and with the software Le Phare with the same input parameters as ours (once considering our adaptations to a $z=1.26$ sample). When using Ilbert et al. (2009) photometric redshifts as reference redshifts (hereafter $z_{\text {phot-COSMOs), }}$ ), calibrated on VIMOS/VLT and DEIMOS/Keck spectroscopy, and estimating photometric redshift for the COSMOS sample using only the four bandpasses we use in this paper (hereafter $z_{\text {phot-4b }}$ ), we obtain a difference in photometric redshifts $\left(z_{\text {phot }-4 \mathrm{~b}}-z_{\text {phot-COSMOS }}\right)$ consistent with zero, with a standard deviation $\sigma=0.12$. Ilbert et al. (2009) estimate the uncertainty on their photometric redshifts to be $\sigma_{z} \approx 0.03$, at $z=1.26$ and at the magnitude depth of our sample. We estimate then the uncertainty on our photometric redshift measurement to be $\sigma_{\mathrm{opz}}=0.12$. When we estimate how many COSMOS galaxies with $z_{\text {phot-COSMOS }}$ in the range $1.26 \pm 3 \sigma_{\mathrm{opz}}$ are recovered within the range $1.26 \pm 3 \sigma_{\mathrm{opz}}$ in $z_{\text {phot }-4 \mathrm{~b}}$, we find $87 \%$ of the objects. On the other hand, when estimating how many objects with $z_{\text {phot}-4 b}$ in the range $1.26 \pm 3 \sigma_{\mathrm{opz}}$, also have $z_{\text {phot-COSMOS }}$ in that range, we obtain $85 \%$. When instead of $1.26 \pm 3 \sigma$, we use our empirical selection, e.g., the range in redshift $0.92<z_{\text {phot }}<1.36$, these percentages become $96 \%$ and $87 \%$, very similar to the percentage of completeness and purity, respectively, which we have estimated based on our empirical analysis. These values are 
also compatible with more sophisticated estimates of uncertainties on photometric redshift and group membership estimated in George et al. (2011).

Therefore, we estimate our photometric redshift uncertainty to be $\sigma=0.12$ at $z=1.26$, and that our photometric redshift selection is $\approx 95 \%$ complete for the early-type galaxies $(\approx 87 \%$ for the late type), with a contamination of $\approx 20 \%$.

To further select group members, we applied an FoF algorithm. As starting center we initially used the overdensity centers defined by Nakata et al. (2005). When galaxies have morphological classifications (see below), we redefine our final group centers as luminosity-weighted centers from our early-type red population. Our linking scale was normalized on the two main clusters and chosen as the scale at which we select all spectroscopically confirmed cluster members. We obtain a linking scale corresponding to a local distance of $0.54 \mathrm{Mpc}$, normalized to $z=1.26$ and to our magnitude range as in Postman et al. (2005).

We have also re-selected the cluster galaxy sample exactly in the same way as the group sample, using photometric redshifts and the ACS GTO spectroscopic follow-up described in Mei et al. (2006b, 2009). Our cluster sample remains unchanged.

Our final sample comprises 89 galaxies in the clusters, of which 41 are spectroscopically confirmed, and 74 galaxies in the groups, of which 25 are spectroscopically confirmed.

\section{MORPHOLOGICAL CLASSIFICATION AND PHOTOMETRY}

\subsection{Morphological Classification}

All our selected group members have been morphologically classified using the same visual classification as Postman et al. (2005), based on the ACS $z_{850}$ images, the closest to the rest-frame $B$ band (conventionally used for morphological classification) at $z=1.26$. Specifically, we classified galaxies as early type (ETGs; defined as elliptical and S0s), late type (Sa, $\mathrm{Sb}$, and Sc), and irregular/undefined. With respect to Postman et al. (2005), we have added a new class of clumpy/uncertain objects and objects presenting interactions or visible tidal tails to study their occurrence in the cluster and group population. We will refer to these objects as disturbed. Three of us, S.M., M.P. and A.S., have independently classified all galaxies in this sample and compared our results. As already noted in other work (e.g., Postman et al. 2005), our classification of early-type versus late-type galaxies was the same in around $90 \%$ of the cases, while it is the same in approximately $70 \%$ of the cases when distinguishing the early- (e.g., elliptical from S0) and latetype subclasses. From here on, the terms early-type and latetype galaxies refer, respectively, to galaxies morphologically classified as elliptical and S0, and as spiral galaxies according to this visual classification. Sa galaxies are classified so because they show diffuse features that look like spiral arms around a massive bulge. We call this class of galaxies bulge-dominated spirals (hereafter BDSs or galaxies visual classified as Sa), and we cannot exclude that the diffuse component that we identify as a disk might be tidal structures or merger remnants. We show our new visual classification in Figures 2-4.

For each of the galaxies, we also measured the $180^{\circ}$ rotational asymmetry $A$ and image concentration $C$ parameters (Abraham et al. 1994; Conselice et al. 2000) using the PyCA software (Menanteau et al. 2006). In Figure 5, we show our visual morphology in a concentration/asymmetry plane. Most of our visually classified ETGs have $C>0.3$ and $A<0.1$. In this region, we found $89 \%$ of our total early-type population (excluding the bright early-type pair and triplet-see below), $50 \%$ of the Sa, $13 \%$ of the late spirals, irregulars, or disturbed.

The distribution of the morphological types in this region is $66 \%$ of visual early type, $18 \%$ of Sa galaxies, and $16 \%$ of late-type spirals. We can separate at $10 \%$ early- from late-type galaxies (excluding Sa galaxies) just by simple cuts in the $C / A$ plane, and both samples will have more or less concentrated $\mathrm{Sa}$, around half among the highly concentrated/early-typedominated region and the other half in the less concentrated/ late-type region. Sa galaxies because of their large/concentrated bulges are then a class that highly contaminates the ETGs.

If we draw a line in the $\log (A)$ versus $\log (C)$ plane, following Abraham et al. (1994) and Menanteau et al. (2006), and optimizing it for our sample, we obtain two regions that maximize the recovery of two separate classes of early- and late-type galaxies (e.g., maximizing the maximum percentage of early-type and the less of late-type contamination in the earlytype region). When we use this line to separate classes, we classify as early type $96 \%$ of the visually classified early-type galaxies (ETG), $45 \%$ of the Sa, and $8 \%$ of the late-type spirals. The distribution of the morphological types in this new "earlytype" region is visual early-type $73 \%$, Sa $23 \%$, and late-type $4 \%$. When we exclude Sa galaxies, late-type galaxies are a small contamination for ETGs selected in this way. However, when the entire late-type population is considered, they would contaminate the ETG sample at $\approx 30 \%$, mostly because of the Sa galaxies. This classification would do better than the simple cut above, but we will still have $\approx 30 \%$ of the "early type" that do visually show a disk, and that we visually classify as Sa.

In Figure 5, we also show galaxies with Sérsic index $n>2.5$ (see Section 4.2), often used to separate early- from late-type galaxies. In the combined cluster and group sample, we find that $70 \%$ of the ETGs have $n>2.5$, and $78 \%$ of the late-type galaxies have $n<2.5$. Moreover, $57 \%$ of the galaxies with $n>2.5$ are ETGs and $86 \%$ of the galaxies with $n<2.5$ are late type. If we would classify ETG objects with $n>2.5$, our ETG sample would be contaminated at $\approx 40 \%$ by late-type galaxies.

ETGs identified only by their compactness (or Sérsic index) are highly contaminated by BDSs. Bernardi et al. (2010) obtain similar results in the local universe with a detailed analysis of the local Sloan Digital Sky Survey (SDSS; York et al. 2000) ETG sample.

When using more advanced non-parametric techniques from Huertas-Company et al. $(2008,2009,2011),{ }^{21}$ calibrated on the SDSS visual classifications, they are more efficient and we recover $89 \%$ of our visual ETGs with $5 \%$ contamination (two galaxies which are visually classified as Sas). Interestingly, the algorithm manages also to isolate Sa galaxies with reasonably good accuracy: six of nine visual Sas are classified by the algorithm as early spirals, one as a late spiral, and two as early type.

In this paper, we only use our visual morphological classification and discuss how some of our results would change based on a simple automated classification.

Our first result is an absence of massive/bright red bulge pairs or triplets (dry mergers) in the groups. While we do observe a double red bulge and a triple red bulge merger in the two clusters (see also van Dokkum \& Franx 2001; Yamada et al. 2002; Mei et al. 2006b), no merger of this kind is visible in any of the groups.

\footnotetext{
$\overline{21}$ The code and the training sets are available at http://gepicom04/galsvm.htm.
} 

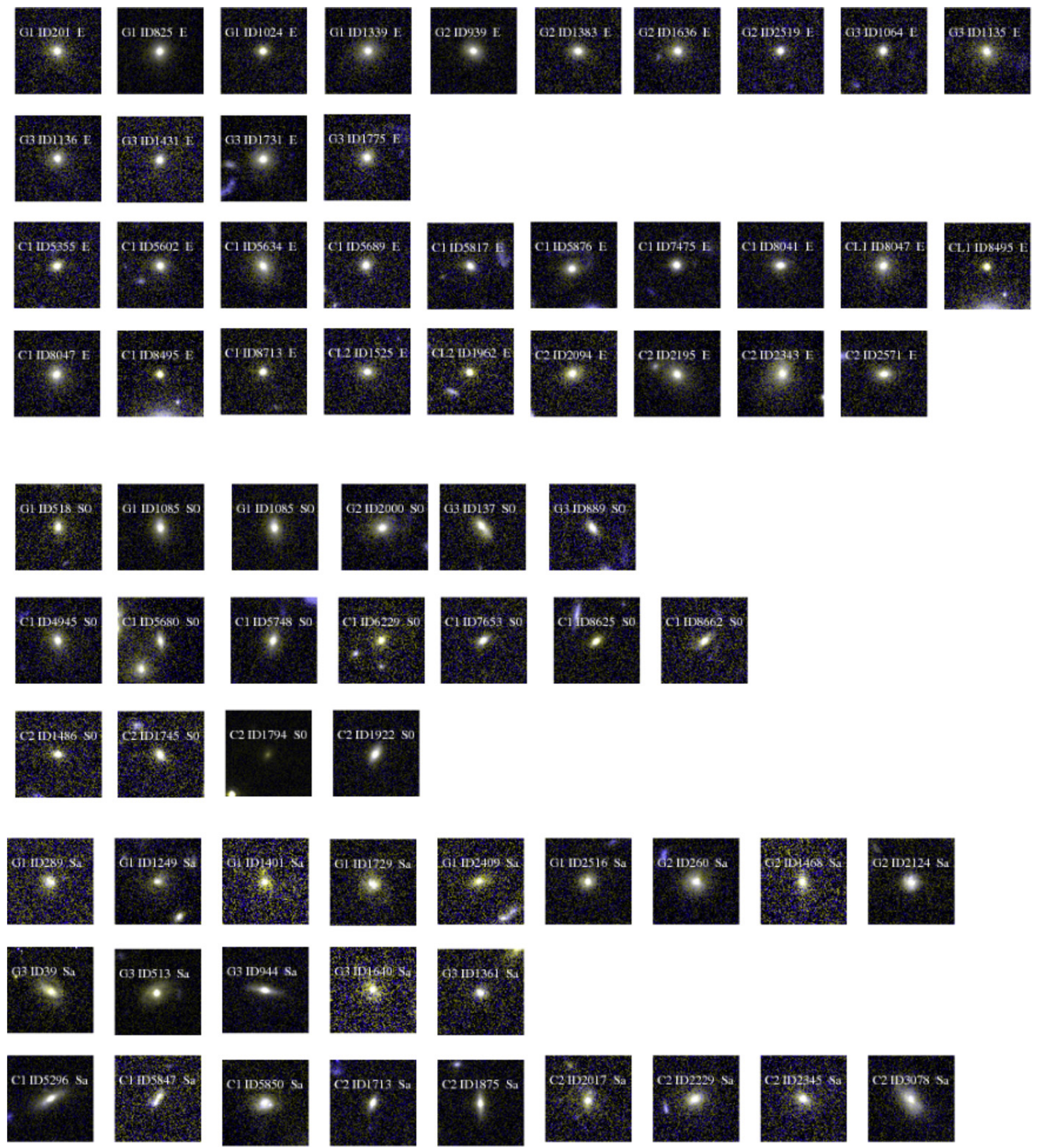

Figure 2. Galaxies classified as elliptical, S0, and Sa (from top to bottom) in the cluster and group sample. The galaxies labeled as $\mathrm{C} 1$ and $\mathrm{C} 2 \mathrm{belong}$ to Lynx E and W, respectively. The galaxies labeled as G1, G2, and G3 belong to Group 1, Group 2, and Group 3, respectively.

(A color version of this figure is available in the online journal.)

Four disturbed objects are observed in the two clusters (three in the less evolved Lynx W) and nine were found in the groups (Figure 4). Disturbed morphologies appear as blue compact galaxies with a companion of the same mass (ID 1282) or smaller (ID 1093), clumpy galaxies, with clumps of similar sizes (9 gal., see Figure 4) or with a central bulge and smaller clumps (ID 910, ID 2043), or as clumps/bulges with large tidal tails (ID 2635, ID 1794). This confirms that galaxies in groups are going through transformations, driven in part by wet/blue late-type mergers, or smaller companion. Most of them show clumpiness, with clumps of similar sizes. These transformations are rarely seen in Lynx W (1), while Lynx E shows three clumpy galaxies.

These observations indicate that wet merger activity is present in both clusters and groups and dry merger activity is only present in the two clusters. Clearly though, since we are studying 

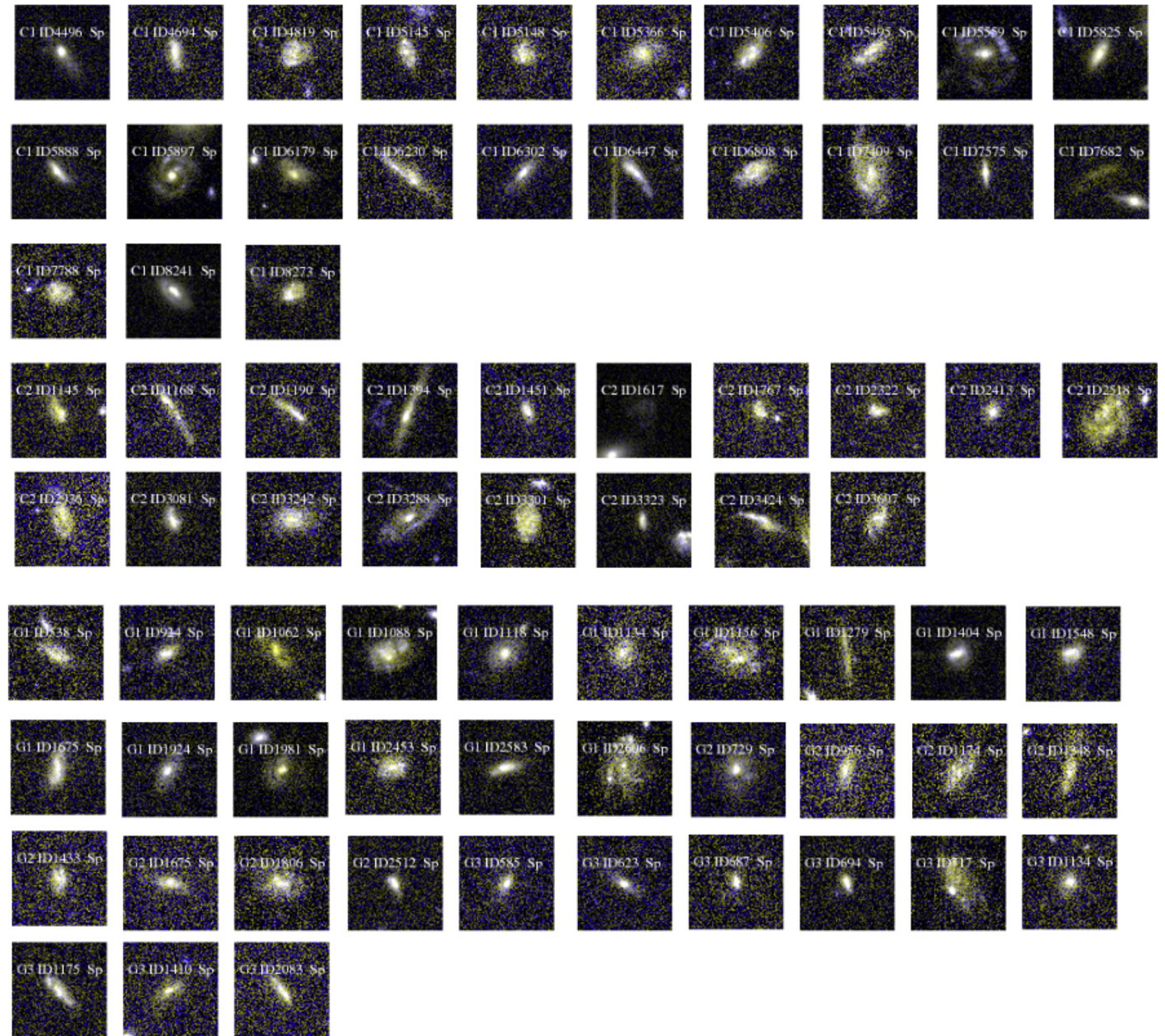

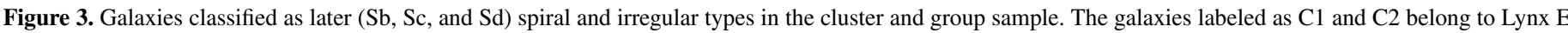
and W, respectively. The galaxies labeled as G1, G2, and G3 belong to Group 1, Group 2, and Group 3, respectively. (A color version of this figure is available in the online journal.)
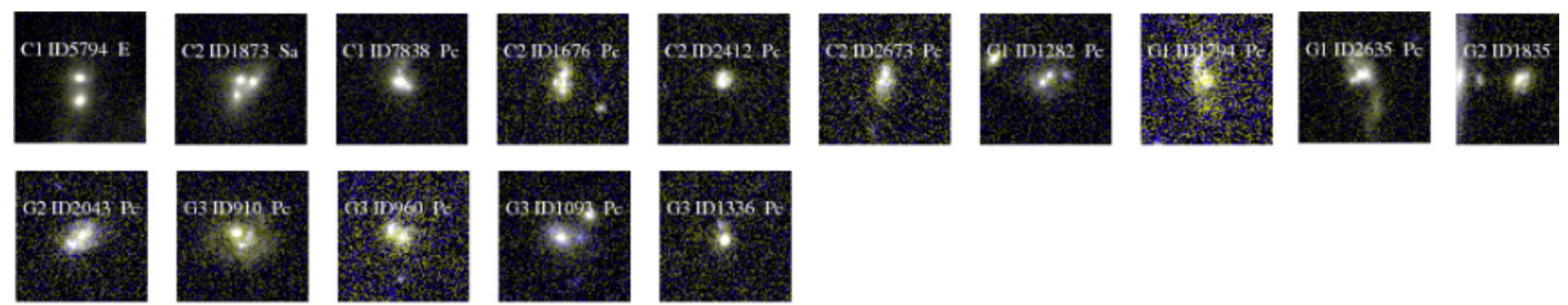

Figure 4. Galaxies classified as mergers or peculiar in the cluster and group sample. The galaxies labeled as C1 and C2 belong to Lynx E and W, respectively. The galaxies labeled as G1, G2, and G3 belong to Group 1, Group 2, and Group 3, respectively.

(A color version of this figure is available in the online journal.)

a single supercluster, these results are not statistically significant (as in the case of other work at these high redshifts) and should be confirmed by larger statistical samples of superclusters of galaxies at these redshifts, unfortunately unavailable at this time (e.g., at present, this is the best we can do).
In Figure 6, we show the spatial distribution of our selected sample, the morphological types, and the red $-0.8<\left(i_{775}-\right.$ $\left.z_{850}\right)<1.2-$ overdensities in the clusters and the groups. Group 1 is very close to Lynx $\mathrm{W}$, and its galaxies are connected by an FoF algorithm. We consider them as two separate structures, 


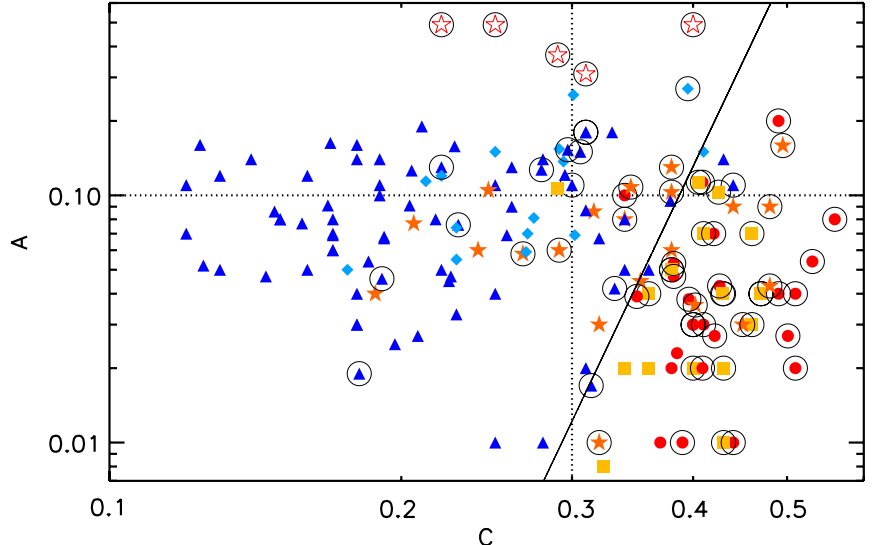

Figure 5. Our visual morphological classification in the Concentration $(C)$ vs. Asymmetry (A) plane. Elliptical, S0, Sa, and late spirals are shown, respectively, as red circles, yellow squares, orange stars, and blue triangles. Clumpy/undefined and late-type merger (e.g., disturbed) galaxies are shown as light blue diamonds and bulge mergers as red open stars. Most of our earlytype population has $C<0.3$ and $A<0.1$ (dotted lines; see the text for more details). If the galaxies were only automatically classified, though, compact and symmetric galaxies $(C<0.3$ and $A<0.1)$ would include around half of the Sa population and $\approx 10 \%$ of the later type spirals, for a total contamination of the early-type sample of $\approx 37 \%$. The continuous line maximizes the recovery of the two separate early- and late-type classes. When using this line to separate classes, we classify as early type $96 \%$ of the visually classified early-type galaxies, $42 \%$ of the Sa, and $7 \%$ of the late-type spirals. The distribution of the morphological types in this new "early-type" region is visual early type $73 \%$, Sa $23 \%$, and late type $4 \%$. Late-type galaxies would contaminate the early-type sample at $\approx 30 \%$, mostly because Sa galaxies would be classified as early type. Empty circles show galaxies with $n>2.5$, often used to separate early- from late-type galaxies. Had we used this criteria to define our early-type sample, we would have suffered a contamination of $\sim 40 \%$, especially from Sa galaxies.

(A color version of this figure is available in the online journal.)

nevertheless, because the center of the group lies at $\sim 1.1 R_{200}$ from the center of the cluster and extends to $\sim 2 R_{200}$, with an area of very low density between 0.5 and $1 \sim R_{200}$. It might be close to merging, or in the merger process. High-resolution $\mathrm{X}$-ray imaging would give us more elements to understand the nature of their interaction.

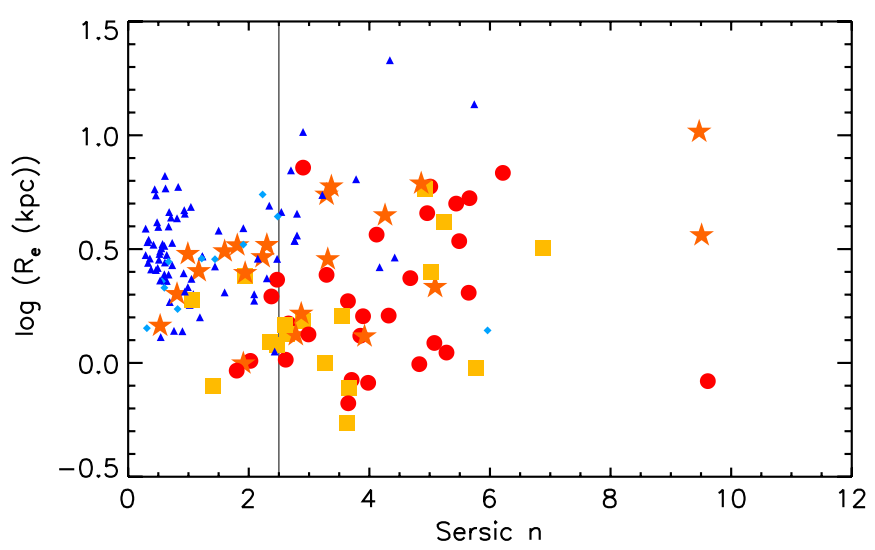

Figure 7. Size as a function of Sérsic index $n$ for all morphological types. Symbols are the same as in Figure 5. The continuous vertical line shows $n=2.5$ often used to separate early- from late-type galaxies (see caption of Figure 5 for discussion). Most of our galaxies have $n<6$. In some cases, we find larger Sérsic indexes, but we do not observe any indication of bias in the size estimation (e.g., we do not see a correlation between $n$ and size). Symbols for late-type galaxies are smaller because of the high density of this kind of galaxies at $n<1$. (A color version of this figure is available in the online journal.)

\subsection{Structural Parameters, Color, and Magnitude Measurement}

We derived galaxy structural parameters using GALFIT (Peng et al. 2002) in the ACS $z_{850}$ imaging, fitting elliptical Sérsic models to each galaxy image. With respect to our previous work (e.g., Mei et al. 2009), in this work we did not constrain the values of the Sérsic index for most of the sample, as suggested in Peng et al. (2002). Only the five galaxies found in double or triplets in the clusters required simultaneous fitting and a constraint $n<4$ in order for the fit to converge. For each galaxy, we obtain its ellipticity (defined as $1-q$, where $q=(b / a)$ is the axial ratio), its average half-light radius $R_{e}$, and Sérsic index $n$. In Figure 7, we plot size as a function of the Sérsic index $n$ for our different morphological types. While most galaxies have Sérsic index $n<6$, for some the fit converged to higher values

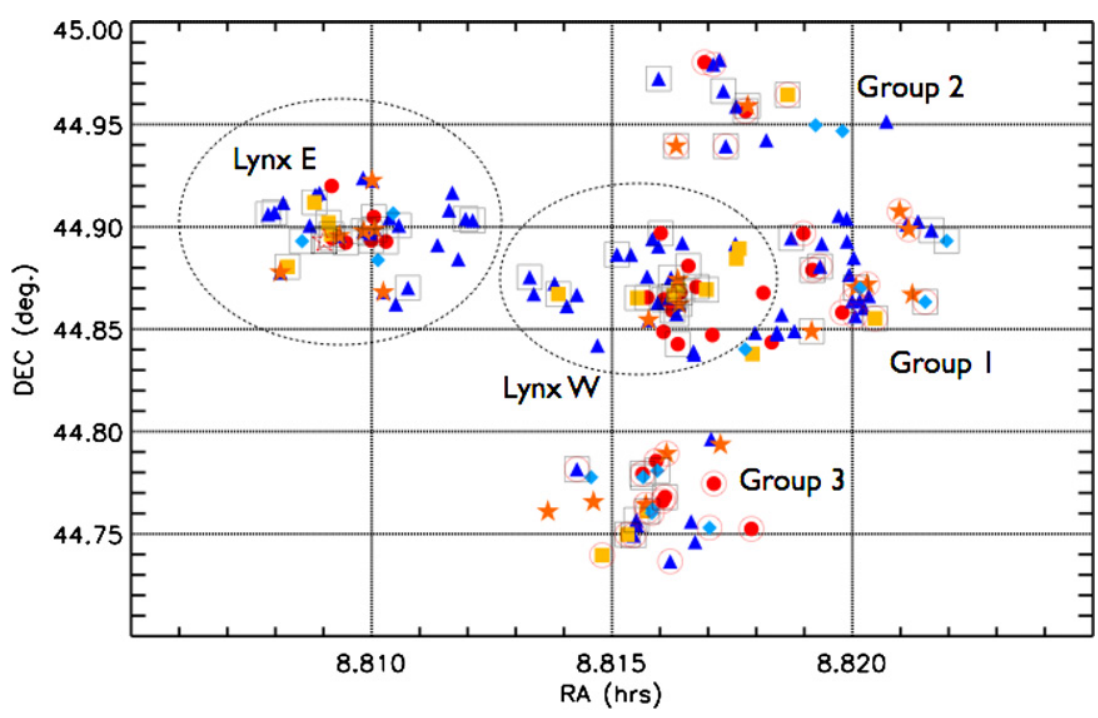

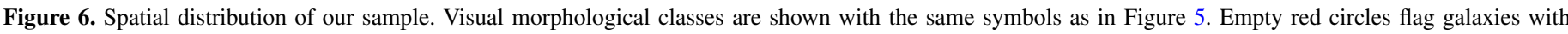

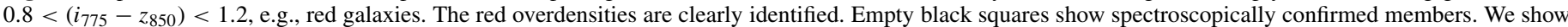

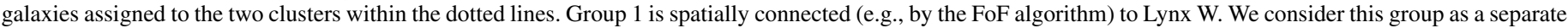

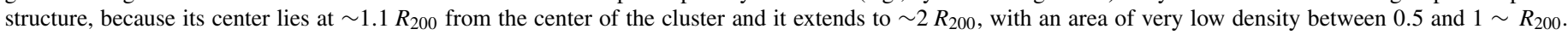
Group 1 might be close to merging with Lynx $\mathrm{W}$ or in the merger process.

(A color version of this figure is available in the online journal.) 
of $n$. In these latter cases, we do not see any evidence for a bias in size estimation. To see if there is a bias in size estimation, e.g., a correlation between $n$ and $R_{e}$, we calculate Pearson coefficients and the probability of correlation between these two variables. We obtained a probability of correlation $<60 \%$ and a probability to have a random distribution $>95 \%$, for both the entire sample and for the early- and late-type populations separately.

We measured galaxy colors following Mei et al. (2006a, 2006b; see also Blakeslee et al. 2006). Our colors were measured inside a circular aperture scaled by the galaxy average half-light radius $R_{e} \sqrt{q}$ (van Dokkum et al. 1998, 2000; Scodeggio 2001) to avoid galaxy gradient effects.

For ETGs, we have checked (Mei et al. 2006a) that our colors do not change (within the uncertainties) if the effective radii are calculated via a two-component (Sérsic bulge + exponential disk) surface brightness decomposition technique using GIM2D (Marleau \& Simard 1998; Rettura et al. 2006) that better fits the galaxy light profiles. For late-type galaxies, we used a single profile decomposition, so that their aperture radius would be larger than their bulge, but this does not take into account the extension of their disk. For early-type spirals (Sas), this value is very close to their bulge size.

We estimate uncertainties on the color by adding the uncertainty due to flat fielding, PSF variations, and ACS pixel-topixel correlations in quadrature to the flux uncertainties (Sirianni et al. 2005). Our average color uncertainties range from 0.01 to $0.03 \mathrm{mag}$.

As an estimate of galaxy total magnitude, we used SExtractor's MAGAUTO. This quantity is not a perfect estimator (see discussion in Mei et al. 2009 and references therein), which must be borne in mind when comparing to other samples.

\section{RESULTS}

\subsection{Morphology-Density Relation}

Morphology-density relations in the Lynx clusters and groups, as derived from our visual morphological classification, are shown in Figures 8 and 9. Here, we have counted earlytype mergers as early type, and late-type mergers or unknown morphologies as late type. Projected densities were estimated following Postman et al. (2005). We used a seven nearest neighbor algorithm with a $f_{\text {corr }}=3$. To correct for background contamination, we subtract from galaxy type counts an estimated number of non-cluster members, as $N_{T}^{c}=N_{\text {total }} f_{\text {total,c }} f_{c, T}$ (see Postman et al., Appendix B for details). $N_{\text {total }}$ is the total number of galaxies in a density bin, $f_{\text {total,c }}$ the total number of contaminants, estimated from our spectroscopic sample with the new photometric redshifts, and $f_{c, T}$ the contamination rate expected for each morphological class.

Figure 8 shows the visual early- (E, S0) and late-type fractions. In the two clusters, the early-type fraction increases with density, as expected in galaxy clusters. However, the earlytype fraction in the two Lynx clusters never increases beyond $\approx 50 \%$, as compared to the $\approx 80 \%$ observed in massive clusters at $z \approx 1$ (Postman et al. 2005). In the top panel of Figure 8, we show Postman et al. (2005) results for the entire seven-cluster sample from the ACS IRCS. Compared to the entire sample, the early fraction in the Lynx clusters shows a further lack of early-type galaxies, with a confidence of $\sim 2 \sigma$. This is mainly due to a strong presence of late-type galaxies in the Lynx W sample. Note that Lynx W was not used in the Postman et al. (2005) analysis.

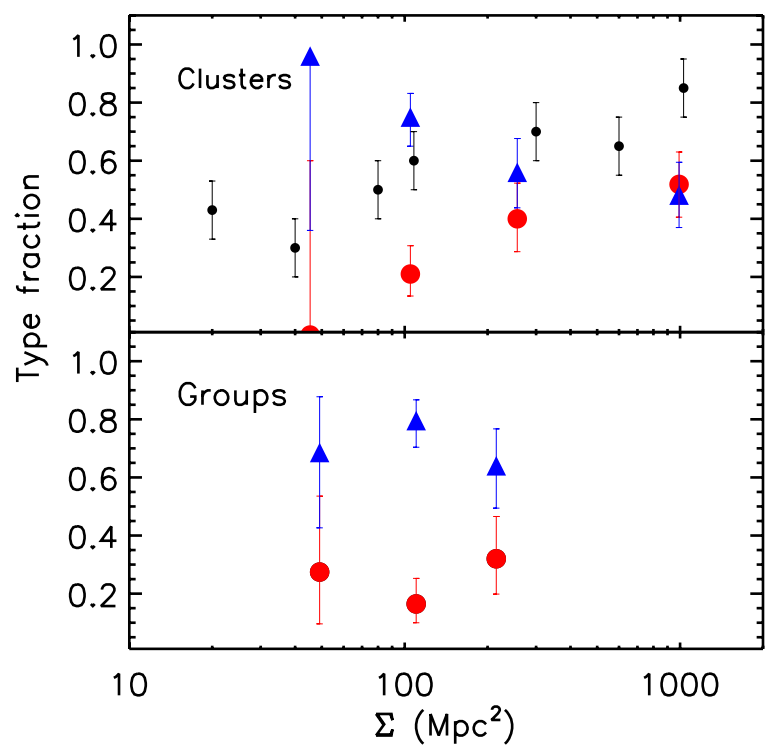

Figure 8. Morphology-density relation in the Lynx clusters (top) and groups (bottom) for our luminosity-limited sample. We use visual morphology to separate ETGs from late-type galaxies. We show early-type (red circles) and late-type (blue triangles) fractions as a function of projected galaxy density (see the text and note that these symbols are different from those used in Figure 5). In the two clusters we observe an increase in early-type fraction with density, as expected in galaxy clusters, with a difference at the high-density end. As a comparison, we show ETG fractions from the entire seven-cluster sample from the ACS Intermediate cluster survey (Postman et al. 2005) as small black dots. The entire sample shows an increase of the early-type population up to $\approx 80 \%$. In the Lynx clusters, however, the early-type fraction does not exceed the late-type population fraction. In fact, they are found to contribute half of the entire population even at high densities. The group fractions are dominated by late-type galaxies, with fractions of early types never exceeding $\approx 25 \%$ of the sample, a value consistent with field fractions and Lynx cluster fractions at the same densities (see the top figure).

(A color version of this figure is available in the online journal.)

The group populations are dominated by late-type galaxies, with the fraction of early types never exceeding $\sim 25 \%$ of the sample, a fraction consistent with that in the two Lynx clusters in regions of the same density as the groups and similar to field fractions at these redshifts (e.g., Figure 9 in Postman et al. 2005). Part of this is due to an overdensity of late-type galaxies in Group 1 (see Figure 6, top right of Group 1). We have verified that galaxy fractions do not change significantly even when this overdensity is excluded from the analysis, with early-type fractions remaining close to $\sim 25 \%$.

Since we cannot exclude that diffuse features around $\mathrm{Sa}$ galaxies might be tidal structures or merger remnants (instead of a disk), we estimated fractions of what we call bulge-dominated galaxies (hereafter BDGs): visual ETGs, Es and S0s, plus BDSs (BDSs are the visual Sas by definition). In this way we want to verify if, when added to the ETGs, the galaxies that we classify as Sas might be the galaxies we are missing when counting only ETGs. In Figure 9, we show the fraction of BDGs and later-type (e.g., not counting the Sas) galaxies. When the entire BDG population is shown, its fraction in the clusters increases to that expected from the entire Postman et al. (2005) sample. In the groups, BDG fractions contribute at $\approx 50 \%$, more consistent with ETG fractions observed in groups at these redshifts (e.g., Poggianti et al. 2009; Just et al. 2010).

As observed in Mei et al. (2009), such a high fraction of BDS is not observed in the other clusters of the ACS IRCS. A similar evolution of early-type spirals is found in the COSMOS field (Bundy et al. 2010), where this population increases at $z \approx 1$ for 


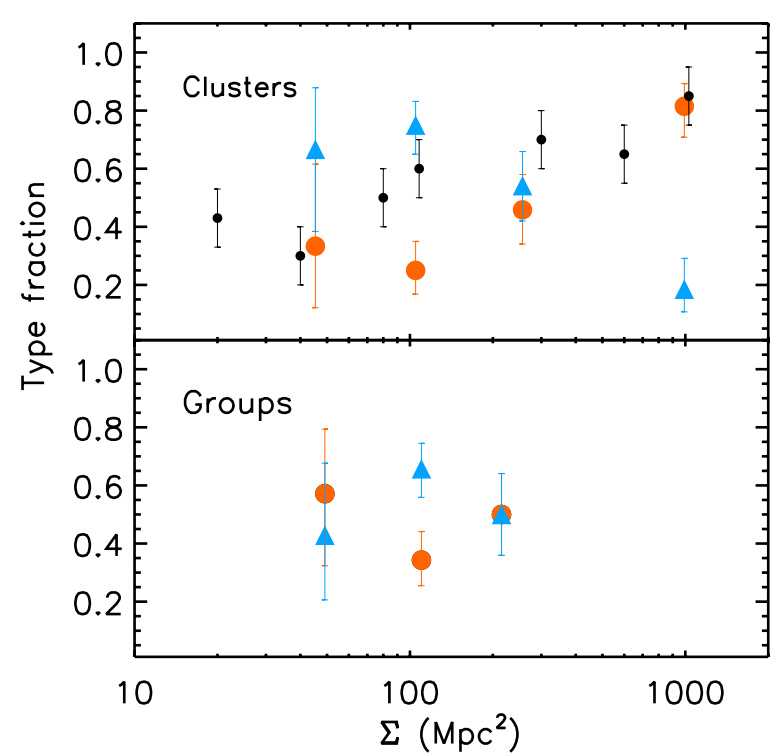

Figure 9. Morphology-density relation for BDGs (orange circles) and late spirals (light blue triangles) for our luminosity-limited sample. In the two clusters, we see an increasing bulge fraction with density. For comparison we show early-type fractions from the entire seven-cluster sample from the ACS Intermediate cluster survey (Postman et al. 2005) as small black dots. Compared to Figure 8, the BDG population fraction in the clusters approaches that ETG fraction observed in the entire ACS Intermediate cluster survey from Postman et al. (2005). The group fractions show that the BDG and late-type spiral populations contribute each about half of the total population, and are closer to cluster fractions at the same densities (see the top figure) and in agreement with group fractions at these redshifts.

(A color version of this figure is available in the online journal.)

galaxies with masses similar to those of our sample. Since the fraction of early- to late-type galaxies changes between these two clusters and clusters at $z<1.2$, while the number of BDG does not, as pointed out in Mei et al. (2009), these populations, whether they are spiral or ETGs with tidal features or merger remnants, could eventually evolve into the ETG morphologies as we observe them at $z \approx 1$. We might speculate that a similar evolution might have occurred in the groups, but with the small statistics we have, we are unable to address this question.

\subsection{The Color-Magnitude Relation in Groups and Clusters}

The CMRs for the clusters and groups are shown in Figure 10. Two of the bright spirals in the clusters are spectroscopically confirmed; the brightest is in the very center of the Lynx E. The fits to the red sequence have been performed as in Mei et al. (2009) for different early-type populations in the clusters and the groups. We fit three parameters: the zero point, slope, and scatter around the red sequence:

$$
\left(i_{775}-z_{850}\right)=\text { Zero Point }+ \text { Slope } \times\left(z_{850}-22.5\right) .
$$

The CMR was fit using a robust linear fit based on Bisquare weights (Tukey's biweight; Press et al. 1992), and the uncertainties on the fit coefficients were obtained by bootstrapping on 1000 simulations. The scatter around the fit was estimated from a biweight scale estimator (Beers et al. 1990) that is insensitive to outliers in the same set of bootstrap simulations. A linear, least-squares fit with $3 \sigma$ clipping and standard rms scatter gives similar results to the biweight scale estimator within $\approx 0.001-0.002 \mathrm{mag}$ for the slope and the scatter.

To estimate the intrinsic galaxy scatter (i.e., not due to galaxy color measurement uncertainties), we estimated the additional scatter needed beyond the measurement error to make the observed $\chi^{2}$ per degree of freedom of the fit equal to one. Again, the uncertainty on the internal scatter was calculated by bootstrapping on 1000 simulations.

Our results are given in Tables 2 and 3, for all cluster and group-selected galaxies and for the galaxies within one virial radius, respectively. The continuous lines in Figure 10 show the fit to the cluster elliptical red sequence within one virial radius. The dashed lines show the fit and the $3 \sigma$ scatter around the elliptical red sequence in the groups, within one virial radius.

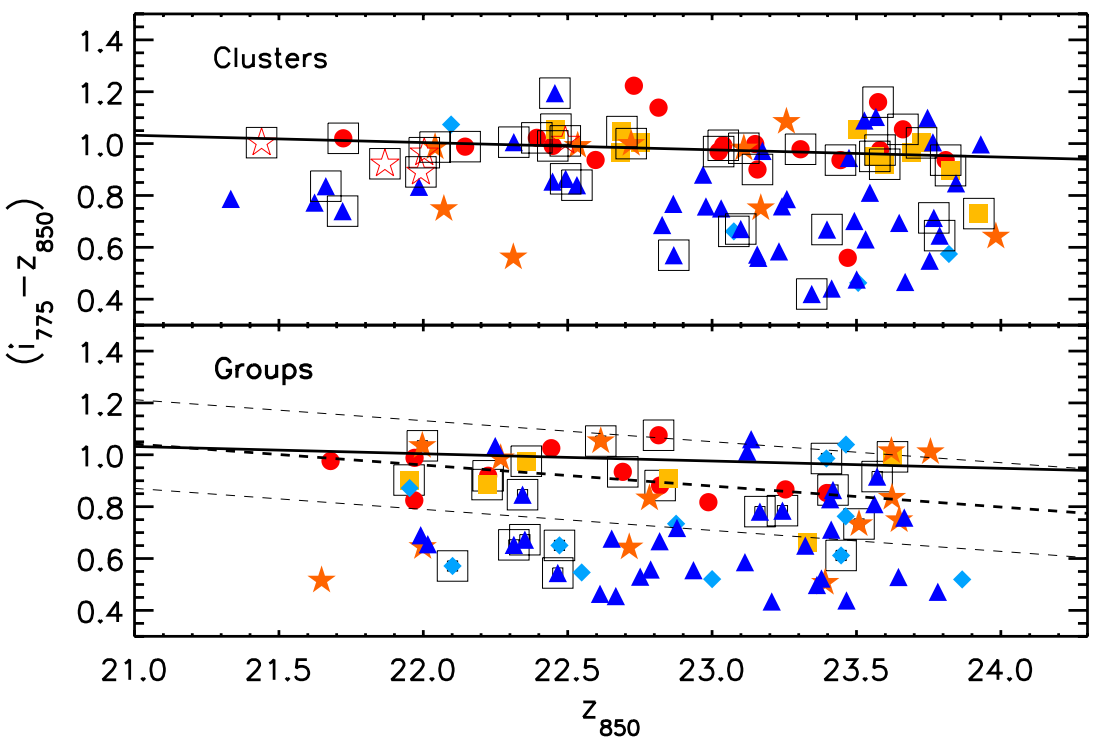

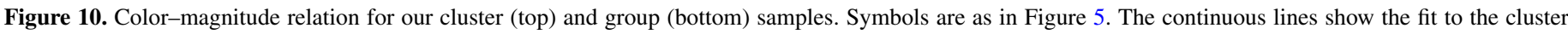

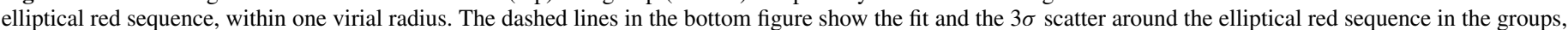

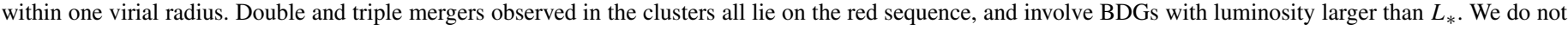

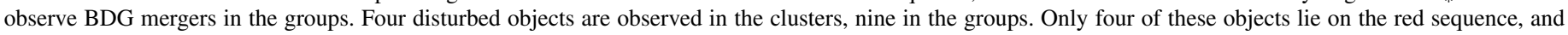
their luminosity is equally distributed between high and low luminosities.

(A color version of this figure is available in the online journal.) 
Table 2

CMR Fit Results for All Our Selected Galaxies

\begin{tabular}{lcrcrc}
\hline \hline Sample & Type & $N^{\text {gal }}$ & $\begin{array}{c}\text { Zero Point } \\
(\mathrm{mag})\end{array}$ & Slope & $\begin{array}{c}\text { Scatter } \\
(\mathrm{mag})\end{array}$ \\
\hline Clusters & E+S0 & 26 & $1.01 \pm 0.01$ & $-0.027 \pm 0.019$ & $0.058 \pm 0.012$ \\
& E & 16 & $0.99 \pm 0.01$ & $-0.014 \pm 0.028$ & $0.065 \pm 0.020$ \\
& S0 & 10 & $1.03 \pm 0.02$ & $-0.060 \pm 0.031$ & $0.036 \pm 0.013$ \\
Lynx E & E+S0 & 19 & $1.00 \pm 0.02$ & $0.001 \pm 0.032$ & $0.063 \pm 0.014$ \\
& E & 12 & $0.99 \pm 0.02$ & $0.023 \pm 0.033$ & $0.064 \pm 0.018$ \\
& S0 & 7 & $1.00 \pm 0.03$ & $-0.020 \pm 0.041$ & $0.037 \pm 0.016$ \\
Lynx W & E+S0 & 9 & $1.00 \pm 0.02$ & $-0.056 \pm 0.018$ & $0.028 \pm 0.015$ \\
& E & 6 & $0.98 \pm 0.02$ & $-0.046 \pm 0.034$ & $0.025 \pm 0.023$ \\
& S0 & 3 & $1.05 \pm 0.01$ & $-0.139 \pm 0.054$ & $0.011 \pm 0.021$ \\
Groups & E+S0 & 15 & $0.92 \pm 0.02$ & $-0.019 \pm 0.050$ & $0.078 \pm 0.010$ \\
& E & 11 & $0.93 \pm 0.04$ & $-0.096 \pm 0.072$ & $0.074 \pm 0.019$ \\
& S0 & 4 & $0.95 \pm 0.01$ & $0.073 \pm 0.013$ & $0.029 \pm 0.026$ \\
\hline
\end{tabular}

For the group ETG red sequence, we found lower zero points (at $\sim 2 \sigma$ ), and larger scatters, both indicating a younger galaxy population (e.g., Kodama \& Arimoto 1997; Kauffman \& Charlot 1998; Bernardi et al. 2005; Gallazzi et al. 2006).

To quantify these differences, we use predictions from stellar population models (e.g., Mei et al. 2009). In Paper II, we demonstrated that galaxy age and mass predictions change significantly for galaxies with ages around 1-2 Gyr, when using stellar population models in which the contribution of the thermally pulsing asymptotic giant branch (TP-AGB) phase is better modeled (Maraston 2005, (M05); Charlot \& Bruzual 2007, in preparation (CB07)). Since Lynx galaxies lie at an epoch in which the universe is $\approx 5 \mathrm{Gyr}$ old, and we have shown the ETGs to have formation epochs between 1-4 Gyr (Papers II and III), it is important that we quantify differences in ages with both BC03 and models with a better accounting of the TP-AGB, in order to test the stability of our results.

Using simple BC03 and CB07 (similar to M05) stellar population models, we find similar results. As in Mei et al. (2009), we consider three cases for each set of models: (1) a simple, single burst solar metallicity stellar population model; (2) a model with solar metallicity and constant star formation rate over a time interval $t_{1}-t_{2}$, randomly chosen to lie between the age of the cluster and the recombination epoch; and (3) a model with solar metallicity and with an exponentially decaying star formation rate. Models (1), (2), and (3) yield similar results, as already noted in Mei et al. (2009).

With both the single burst and the constant star formation rate model, we find the average luminosity-weighted age for ellipticals and ETGs in the groups to be $\sim 0.5-0.7$ Gyr younger than the cluster ellipticals, similar to peripheral ellipticals, and S0 galaxies in the two clusters (Mei et al. 2009). A model with solar metallicity and an exponentially decaying star formation rate, however, predicts that if galaxies with larger scatters had a different star formation history (exponential decay versus single burst), they would have an average luminosity-weighted age similar to the cluster ellipticals. This scenario also predicts bluer color residuals, as observed in our groups.

Galaxies in the groups have then either formed in a short episode of star formation (approximated here as a single burst) later than galaxies in the clusters or formed at the same time, but following a star formation history with longer timescales. Fitting galaxy spectral energy distributions (SEDs) in Paper II, we studied galaxy ages and masses in detail, and showed that discriminating between these two hypotheses depends on the adopted stellar population model (see Figure 9 of that paper).
Table 3

CMR Fit Results within One Virial Radius

\begin{tabular}{lcrrrr}
\hline \hline Sample & Type & $N^{\text {gal }}$ & $\begin{array}{c}\text { Zero Point } \\
(\mathrm{mag})\end{array}$ & Slope & $\begin{array}{c}\text { Scatter } \\
(\mathrm{mag})\end{array}$ \\
\hline Clusters & E+S0 & 23 & $1.00 \pm 0.01$ & $-0.032 \pm 0.016$ & $0.037 \pm 0.008$ \\
& E & 14 & $0.99 \pm 0.01$ & $-0.028 \pm 0.014$ & $0.025 \pm 0.010$ \\
& S0 & 9 & $1.03 \pm 0.02$ & $-0.063 \pm 0.040$ & $0.039 \pm 0.015$ \\
Lynx E & E+S0 & 16 & $0.99 \pm 0.02$ & $-0.001 \pm 0.028$ & $0.036 \pm 0.008$ \\
& E & 10 & $0.97 \pm 0.02$ & $0.012 \pm 0.025$ & $0.033 \pm 0.010$ \\
& S0 & 6 & $1.00 \pm 0.04$ & $-0.014 \pm 0.072$ & $0.045 \pm 0.024$ \\
Lynx W & E+S0 & 9 & $1.00 \pm 0.02$ & $-0.057 \pm 0.020$ & $0.027 \pm 0.015$ \\
& E & 6 & $0.98 \pm 0.02$ & $-0.044 \pm 0.036$ & $0.025 \pm 0.023$ \\
& S0 & 3 & $1.05 \pm 0.01$ & $-0.140 \pm 0.054$ & $0.011 \pm 0.021$ \\
Groups & E+S0 & 8 & $0.92 \pm 0.03$ & $0.016 \pm 0.063$ & $0.066 \pm 0.024$ \\
& E & 5 & $0.92 \pm 0.06$ & $-0.081 \pm 0.106$ & $0.057 \pm 0.030$ \\
& S0 & 3 & $0.95 \pm 0.01$ & $0.066 \pm 0.002$ & $0.011 \pm 0.021$ \\
\hline
\end{tabular}

Using M05 and CB07, we find older populations in the two clusters with respect to the groups and the field, in agreement with results from the CMR. In Paper III, we show how, using BC03 models, we obtain different exponential decaying times in the clusters and the field.

A difference between the age in groups and clusters, if it exists, is small when compared to the differences in age in galaxies of different masses in the same sample, as pointed out and discussed in Paper II.

The double and triple bulge mergers observed in the clusters all lie on the red sequence, and involve galaxies with luminosity larger than the characteristic $L_{*}\left(z_{850} m_{*} \approx 23 \mathrm{mag}\right.$ at $z \approx 1.3$; Mei et al. 2006b). We do not observe red bulge mergers in the groups. Moreover, four disturbed objects are observed in the clusters, and nine in the groups. Only four (one in the clusters and three in the groups) of these objects lie on the red sequence (they show clumps, irregular structure, and are probably reddened by dust), and their luminosity is equally distributed to high and low luminosities.

Luminous red bulge mergers occur in clusters, while both the clusters and the groups show disturbed objects that indicate latetype interactions at all luminosities, and mainly in the blue cloud. These disturbed objects show late-type merging, tidal features, and complex morphology that suggest a higher incidence of galaxy transformations in the groups, in terms of mergers and other galaxy interactions, mainly happening in the blue cloud. The four disturbed objects in the cluster are not all in peripheral regions, but lie mostly at around half of the virial radius.

BDGs have mostly red colors, e.g., they tend to lie on the red sequence. While their red colors might indicate a lack of star formation, it could be also hidden by the presence of dust. We estimated their star formation rate using galaxy SED fitting, following our procedure as described in Paper II. On the red sequence, their star formation rates are always estimated at less than $\approx 25 M_{\odot} \mathrm{yr}^{-1}$ and $18 M_{\odot} \mathrm{yr}^{-1}$, in the clusters and the groups, respectively. One BDG in Lynx W has a star formation rate of $66 M_{\odot} \mathrm{yr}^{-1}$ and lies at $>3 \sigma$ (bluer) than the best fit to the ETG red sequence. In the groups, two BDGs have star formation rates $>100 M_{\odot} \mathrm{yr}^{-1}$, and both lie at $>3 \sigma$ (bluer) than the best fit to the ETG red sequence, and one BDG has a star formation rate of $45 M_{\odot} \mathrm{yr}^{-1}$ and lies at $>2.3 \sigma$ (bluer) than the best fit to the ETG red sequence. Most of them do not have significant detections in our MIPS images, as expected from these estimates. BDGs are therefore mostly passive.

In Figure 11, we plot colors as a function of stellar mass and can define a mass-limited sample. Galaxy stellar masses were 

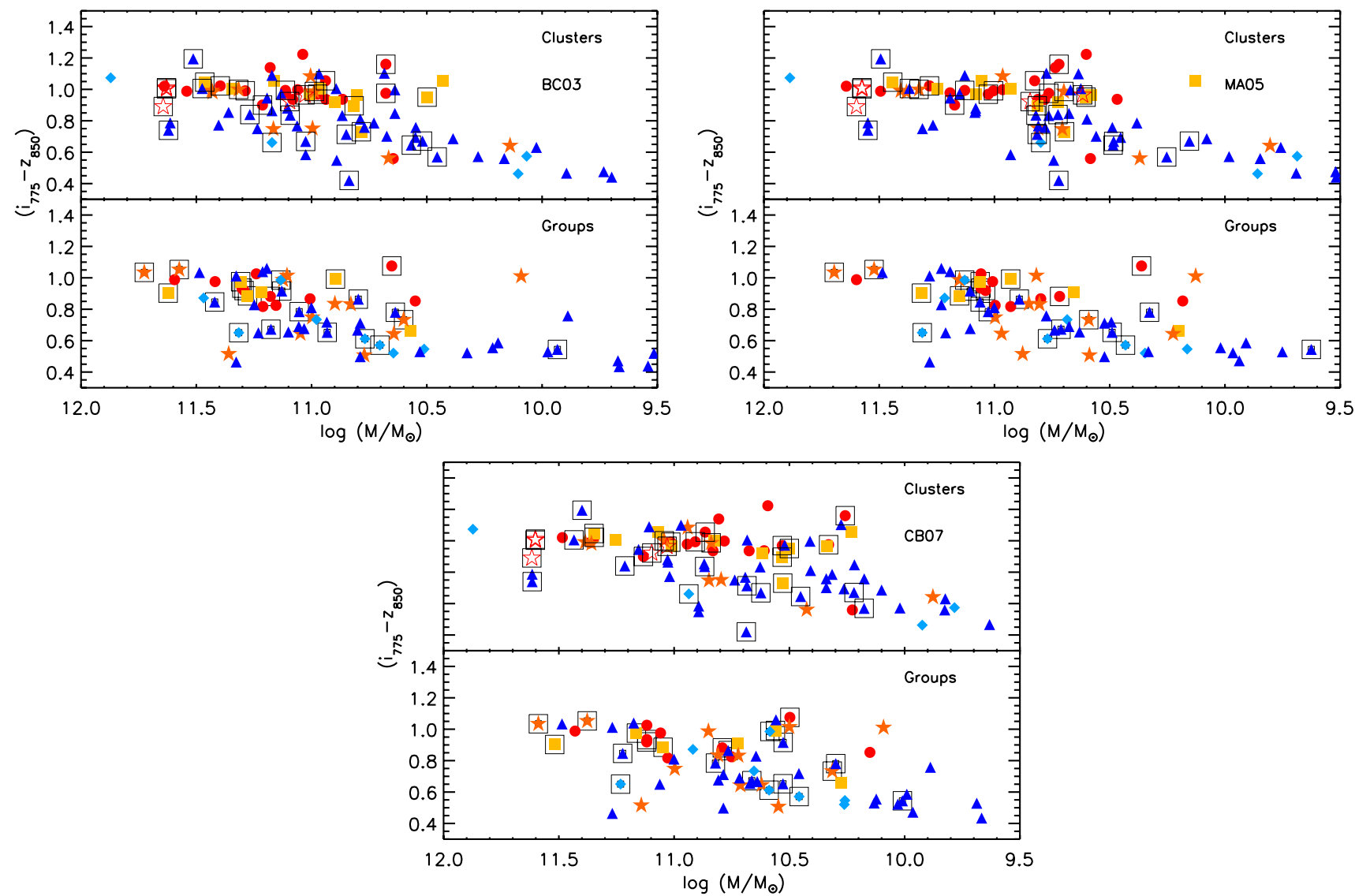

Figure 11. Color-stellar-mass relation for our cluster (top of each panel) and group (bottom of each panel) samples. Symbols are as in Figure 5. Masses are estimated by SED fitting using templates from three stellar population models (BC03, M05, and CB07; see the text for details).

(A color version of this figure is available in the online journal.)

derived by galaxy SED fitting, as described in Paper II, using templates from the three different stellar population models: BC03, M05, and CB07.

As expected, galaxies with the same luminosity range in the color-magnitude diagram have different masses, since the mass-to-light ratio differs between quiescent and star forming galaxies. As discussed in Paper II, ranges in mass are different because we are using different stellar population models: the BC03 model overestimates masses and ages to account for the missing light from the TP-AGB phase (see Paper II for a detailed analysis at our redshift and M05).

If we define mass-limited samples, some of the spiral galaxies that were previously included in the luminosity-limited sample are now excluded. If we use as a conservative lower mass limit in our early-type sample $M=10^{10.6} M_{\odot}, \approx 30 \%(44 \% / 57 \%)$ of the spirals have lower masses when using BC03 (M05/CB07). At a mass limit of $M=10^{10.4} M_{\odot}, \approx 20 \%(30 \% / 39 \%)$ of the spirals have lower masses when using BC03 (M05/CB07).

\subsection{Morphological Content of the Red Sequence}

The morphological content of the red sequence is shown in Figure 12. On the left, we compare early- to late-type fractions in the clusters and groups. On the right, we show the BDG to latertype spiral fraction on the red sequence. As pointed out when analyzing total fractions, ETG fractions on the red sequence are low in the two Lynx clusters when compared to average ETG fractions observed in the other ACS Intermediate Cluster survey clusters at $z<1.2$ (see also Mei et al. 2009). The red sequence in the groups is dominated by late-type galaxies.
Comparing the fraction of BDG and later-type spirals, the BDG fraction is close to the fraction of ETG observed in the other clusters at $z<1.2$, and the groups show results consistent with the ETG fractions in clusters at the same projected density and group fractions at $z \approx 1$ (George et al. 2011). The BDS population in both clusters and groups seems to compensate for the lack of an early-type population. This suggests that at $z \approx 1$, they might evolve into the early-type population on the red sequence, or that they will leave the red sequence later in time.

\subsection{Morphological Fractions in a Mass-limited Sample}

Figure 13 shows early- and late-galaxy fractions in a masslimited sample. We selected galaxies with $M>10^{10.6} M_{\odot}$ to be conservative with respect to the completeness of our ETG sample. The uncertainties on morphological fractions are calculated following Gehrels (1986; see Section 3 for binomial statistics; see also Mei et al. 2009). These approximations apply even when ratios of different events are calculated from small numbers, and yield the lower and upper limits of a binomial distribution within the $84 \%$ confidence limit, corresponding to $1 \sigma$. Note that using this conservative approach our uncertainties are slightly overestimated (Cameron 2011).

In the mass-limited sample, we observe the same trends as in the luminosity-limited sample: the fraction of ETGs is lower than that observed in the rest of the ACS IRCS. BDG fractions, though, show once again fractions that are similar to those of ETGs in the ACS IRCS. Considering galaxies with 


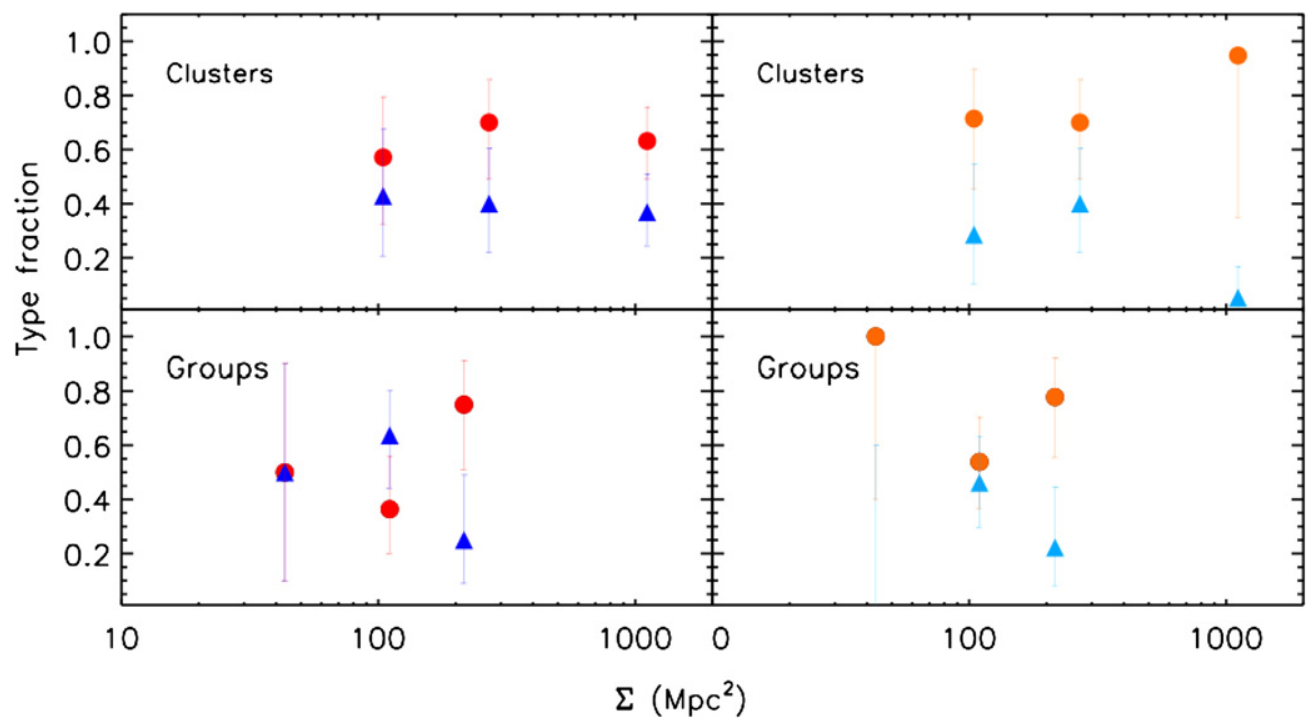

Figure 12. Morphology-density relation on the red sequence. We use the same symbols as in Figures 8 and 9 . On the left, the fraction of early- (red circles) and late-type galaxies (blue triangles) on the red sequence is around 0.5 in groups and low-density cluster regions, in agreement with the results for the entire sample in Figure 8. The percentage of ETGs grows in intermediate density regions. In high-density regions in clusters, though, we again find a fraction of about a half. This is due to the presence of Sa galaxies in these high density regions, three of which are involved in the triple merger in Lynx W. On the right, we show the fraction of BDGs (orange circles) and late spirals (light blue triangles). The BDG fraction in clusters on the red sequence shows the same kind of percentages observed in the overall sample, with BDGs dominating the high-density end. For the groups, the relative fractions of BDG and late spirals are similar to the early- to late-type fractions.

(A color version of this figure is available in the online journal.)

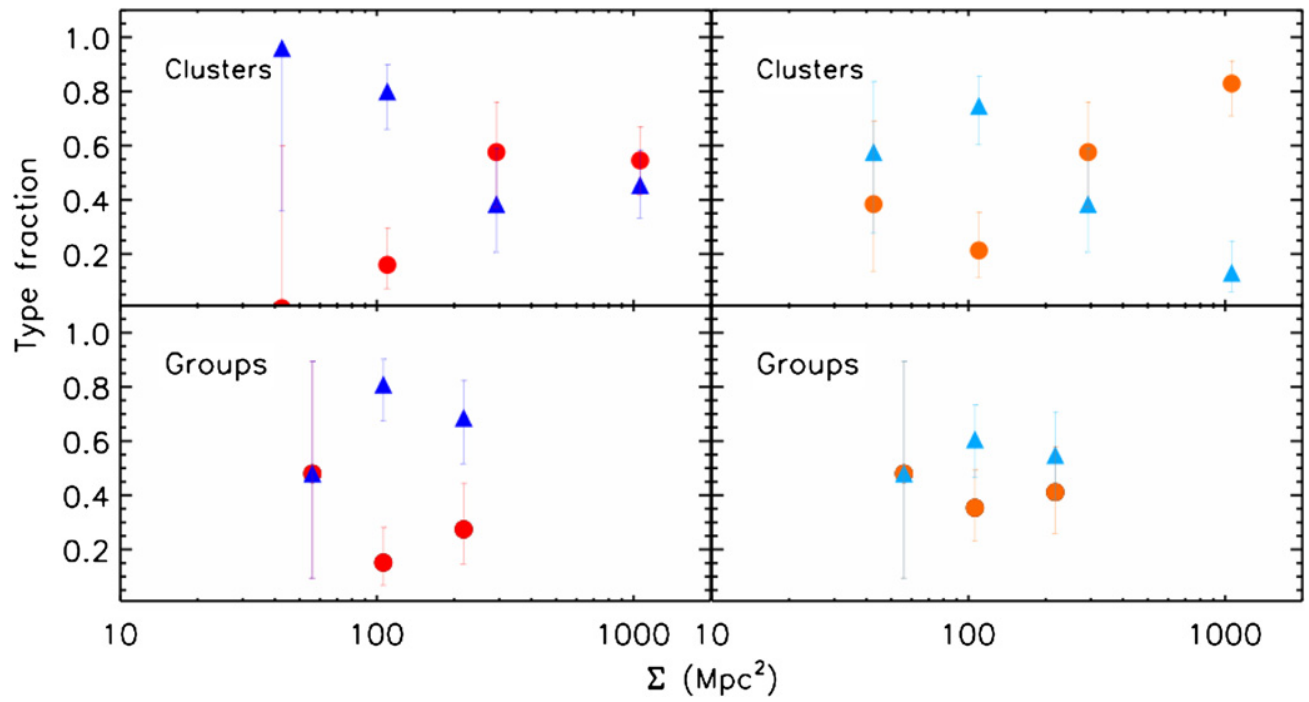

Figure 13. Morphology-density relation in a mass-limited sample, for galaxies with mass $M>10^{10.6} M_{\odot}$. We use the same symbols as in Figures 8 and 9 . On the left, we show the ETG (red circles) and late-type (blue triangles) fractions. On the right, we show the BDG (orange circles) and late spiral (light blue triangles) fractions. Masses are estimated by SED fitting using templates from CB07. Our results do not change significantly using the other two stellar population models.

(A color version of this figure is available in the online journal.)

$M>10^{10.6} M_{\odot}, R<R_{200}$, and projected density $\Sigma$ larger than a given $\Sigma_{\text {lim }}$, we obtain the total early-type/BDG fractions as given in Table 4.

Holden et al. (2007) show that in mass-limited samples, earlytype fractions can change dramatically with respect to those in luminosity-selected samples (e.g., while in both elliptical fractions do not show significant evolution, in luminosityselected samples S0s show evolution). In fact, their work demonstrates that the early-type fraction of galaxies with $M>$ $10^{10.6} M_{\odot}$ does not evolve from $z=0.83$ to the present (opposite to the evolution shown by the luminosity-selected cluster samples from, e.g., Postman et al. 2005; Desai et al. 2007; Poggianti et al. 2009). At $z=0.83$, they report a fraction of $89 \% \pm 7 \%$ for ETGs in regions of density $\Sigma>500 \mathrm{Mpc}^{-2}$, at that mass limit.

Figure 14 compares our results to Holden et al. (2007). In the Lynx clusters, we do observe a lack of ETGs in our mass-limited sample, selected in a manner similar to Holden et al. (2007), with an early-type fraction of $\approx 60 \% \pm 10 \%$ independent of the stellar population model used for mass estimation. However, when estimating the BDG fraction, we obtain $\approx 89 \% \pm 10 \%$, consistent with the ETG fraction found from Holden et al. (2007) at $z=0.83$.

These results are consistent with a lack of a significant evolution in the fractions of ETGs with masses $M>10^{10.6} M_{\odot}$. We obtain similar results for a mass limit of $M>10^{10.4} M_{\odot}$. 
Table 4

Early-type and BDG Fraction for a Mass-limited Sample with $M>10^{10.6} M_{\odot}, R<R_{200}$, and $\Sigma<\Sigma_{\text {lim }}$

\begin{tabular}{|c|c|c|c|c|c|c|c|c|}
\hline Sample & Type & $\Sigma_{\mathrm{lim}}$ & $\begin{array}{c}N_{\mathrm{BC} 03}^{\mathrm{gal}} \\
\left(\mathrm{Mpc}^{-2}\right)\end{array}$ & $\begin{array}{c}\text { Frac. BC03 } \\
(\%)\end{array}$ & $\begin{array}{c}N_{\text {M05 }}^{\mathrm{gal}} \\
(\%)\end{array}$ & $\begin{array}{c}\text { Frac. M05 } \\
\qquad \%)\end{array}$ & $N_{\mathrm{CB} 07}^{\mathrm{gal}}$ & Frac. CB07 \\
\hline \multirow[t]{3}{*}{ Clusters } & $\mathrm{E}+\mathrm{S} 0$ & 80 & 26 & $43 \pm 7$ & 25 & $46 \pm 0.08$ & 20 & $43 \pm 8$ \\
\hline & $\mathrm{E}+\mathrm{S} 0+\mathrm{Sa}$ & 80 & 35 & $57 \pm 7$ & 33 & $60 \pm 7$ & 28 & $60 \pm 8$ \\
\hline & $\mathrm{E}+\mathrm{S} 0+\mathrm{Sa}$ & 500 & 17 & $89 \pm 12$ & 17 & $89_{-13}^{+7}$ & 16 & $89_{-13}^{+7}$ \\
\hline Groups & $\mathrm{E}+\mathrm{S} 0$ & 80 & 13 & $28 \pm 8$ & 12 & $32 \pm 9$ & 11 & $22 \pm 9$ \\
\hline
\end{tabular}

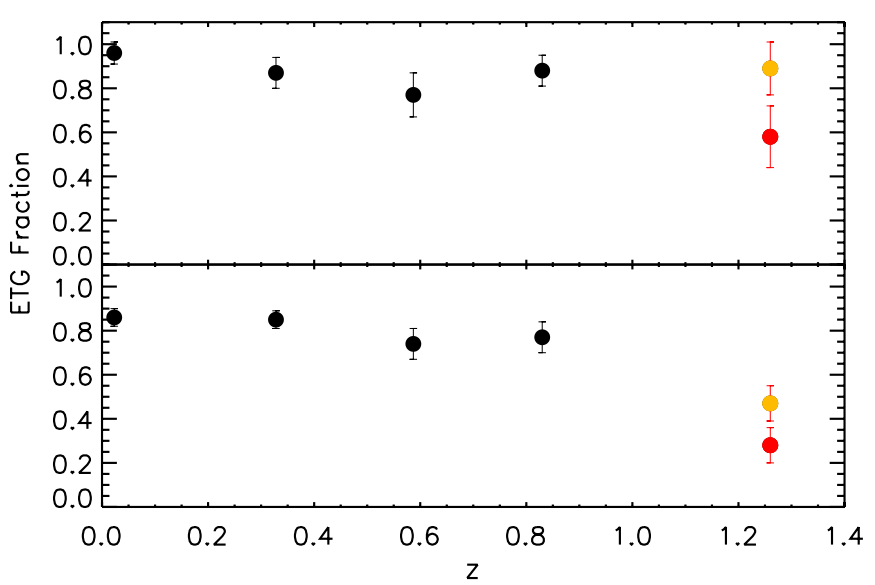

Figure 14. Evolution of the ETG fractions from $z \sim 1.3$ to the present for the clusters (top), and the groups compared to the clusters (bottom). The results from this work (BC03) are compared to those from Holden et al. (2007) for a sample of clusters at $z<1$ (black circles), with galaxies selected in the same way in mass $\left(M>10^{10.6} M_{\odot}\right)$ and in the same density region $\left(\Sigma>500 \mathrm{Mpc}^{-2}\right)$. Lynx ETG and BDG fractions are shown by red and yellow circles, respectively. Cluster ETG fractions do not show a significant evolution up to $z \sim 1$. W. When adding BDS, the results do not change. Group fractions (as expected) are lower than those in the clusters.

(A color version of this figure is available in the online journal.)

We expect our photometric redshift sample to be contaminated at $\sim 20 \%$. Even in the extreme case in which all early types are real cluster members (i.e., no contamination), and $\sim 20 \%$ of the spirals are not, we would obtain fractions of $\approx 65 \% \pm 10 \%$ and $\approx 95 \% \pm 10 \%$ for the cluster ETGs and bulges, respectively.

\subsection{Galaxy Sizes for Different Morphological Types}

The analysis of evolution in morphological fractions has led us to the hypothesis that at $z \approx 1.3$, we observe a BDS population that, losing their disk (or merger remnants or tidal features if these galaxies are not spirals but merger remnants or ETG with tidal features), might have evolved into an ETG population to obtain the ETG fraction observed in clusters at $z<1.2$.

In this scenario, we should also observe a similarity in galaxy size between BDS and ETG galaxies. As we discuss in depth in Paper IV, while field ETGs at $z \approx 1$, selected from the GOODS-CDFS, show a distribution in sizes similar to the local cluster distribution (note this might be different than the field local distribution, we will discuss further this point in future work), Lynx cluster ETGs are more compact than local galaxies at a given mass (refer to Paper IV for the quantitative analysis). These results, consistent with recent results from Valentinuzzi et al. (2010), lead us to conclude that, while we do not need an evolution in the overall mass-size relation for field ETGs, cluster ETGs require an evolution of their mass-size distribution from $z \approx 1$ to the present, in the mass range we probed. As mechanisms of evolution, we propose that either part of the cluster ETGs become larger with time and/or arise from transformations of cluster late-type population (non-ETG progenitors), and/or a new population of larger ETGs is accreted onto the cluster.

In this paper, we explore late-type sizes to understand if part of the larger ETG local population might have evolved from BDSs. In Figure 15, we plot the mass-size relation for our cluster and group sample, compared to the standard SDSS local relation from Shen et al. (2003). In this figure (and hereafter) we use stellar masses derived with $\mathrm{CB} 07$. Our results are the same when using masses derived with BC03 and M05. Shen et al. (2003) use as morphological classification a simple separation in Sérsic index $n$ or compactness. We plot their local relation for early $(n>2.5)$ and late $(n<2.5)$ galaxies and compare them to our results using exactly the same classification (see; note that here we are not using our visual morphological classification as in all other analysis in this paper). We find that neither galaxies in the clusters nor in the groups show evolution in the overall mass-size relation. The mass-size relation in the Lynx superstructure is similar to the Shen et al. (2003) mass-size relation.

However, a simple morphological classification based on one structural parameter is highly contaminated by a mixing of different morphologies, as shown in Section 4, and does not correspond to a visual morphological classification. In Figure 16, we therefore compare galaxies separated into our visual morphological classes (as used in the other analysis in this paper). As a local sample, we use the morphological classified sample from Valentinuzzi et al. (2010) WING clusters, corrected for masses estimated with a Salpeter initial mass function.

When a visual morphological classification is used at both high and low redshifts, ETGs in both clusters and groups show a size distribution more compact than ETGs in the local sample (see Paper IV for a detailed analysis of the ETG Kormendy and mass-size relations in our sample), while spirals do not show evolution in their overall mass-size relation (when their sizes are estimated by a single Sérsic fit).

While our Lynx galaxies do lie within $3 \sigma$ from the average Valentinuzzi et al. (2010) mass-size relation, at a given mass, the two Gaussian distributions are statistically different for masses $M<2 \times 10^{11} M_{\odot}$ which dominate our sample. Using results from Table 1 of Paper IV, and a Kolmogorov-Smirnov and Kuiper statistical test for galaxies with masses $M<$ $2 \times 10^{11} M_{\odot}$, from all three stellar population models, we obtain a probability close to zero that the Valentinuzzi et al. (2010) Gaussian distribution and ours are driven by the same distribution. In the analysis of the ETGs in Paper IV, we concluded that to obtain the local ETG mass-size distribution, the ETG population in clusters and groups must have either accreted larger ETGs, or formed new ones from non-ETG 


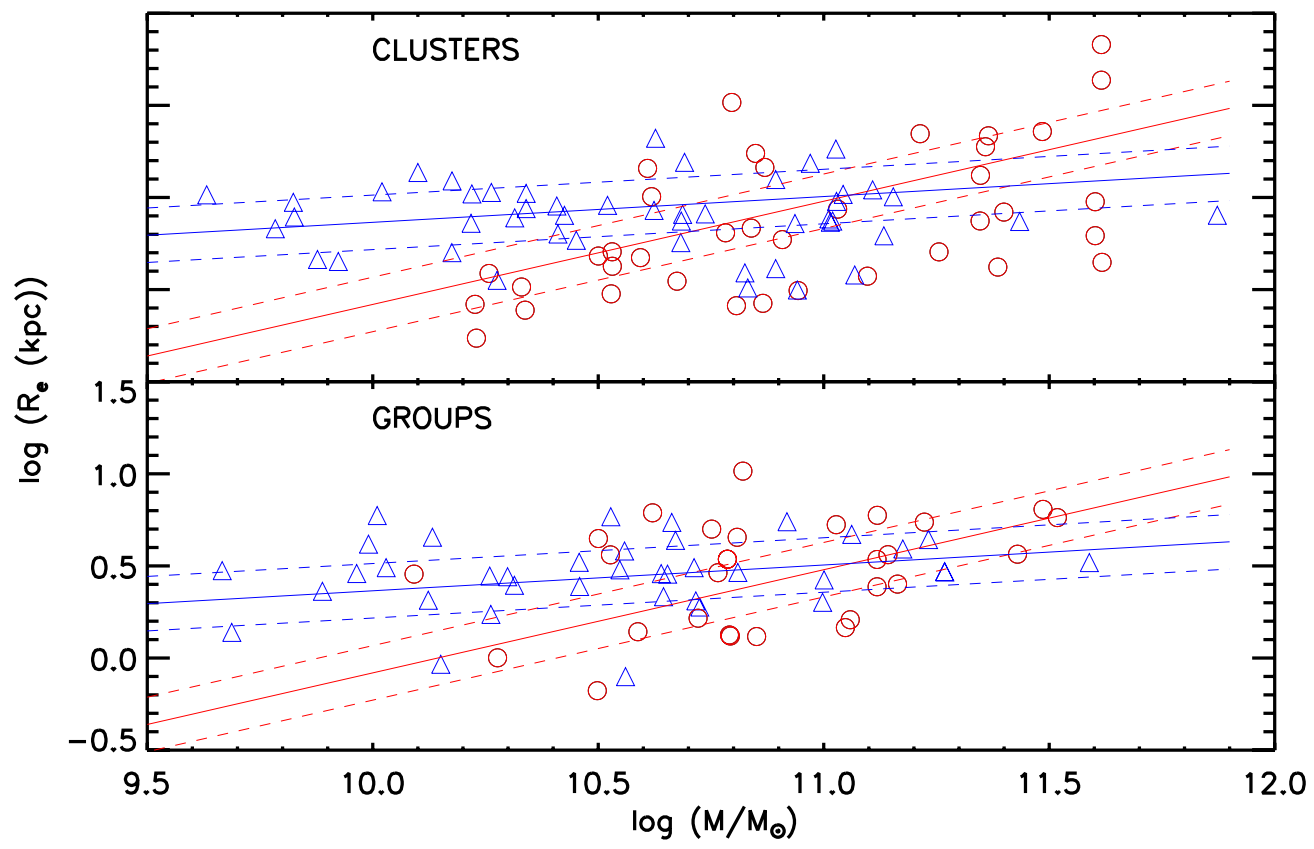

Figure 15. Mass-size relation for all galaxies in our sample (cluster and group galaxies on the top and the bottom, respectively), compared to the local relation from the SDSS. The red (blue) continuous and dashed lines are the Shen et al. (2003) ETG (disk) local relations and their $1 \sigma$ dispersion, respectively. Morphological types in our Lynx sample are selected as in Shen et al. (2003; note that this is not the visual morphological classification we have used so far): red empty circles are galaxies with Sérsic index $n>2.5$ and empty blue triangles are galaxies with $n<2.5$. Using the same morphological class definitions based on Sérsic index shows that both cluster and group galaxy mass-size relations do not evolve.

(A color version of this figure is available in the online journal.)

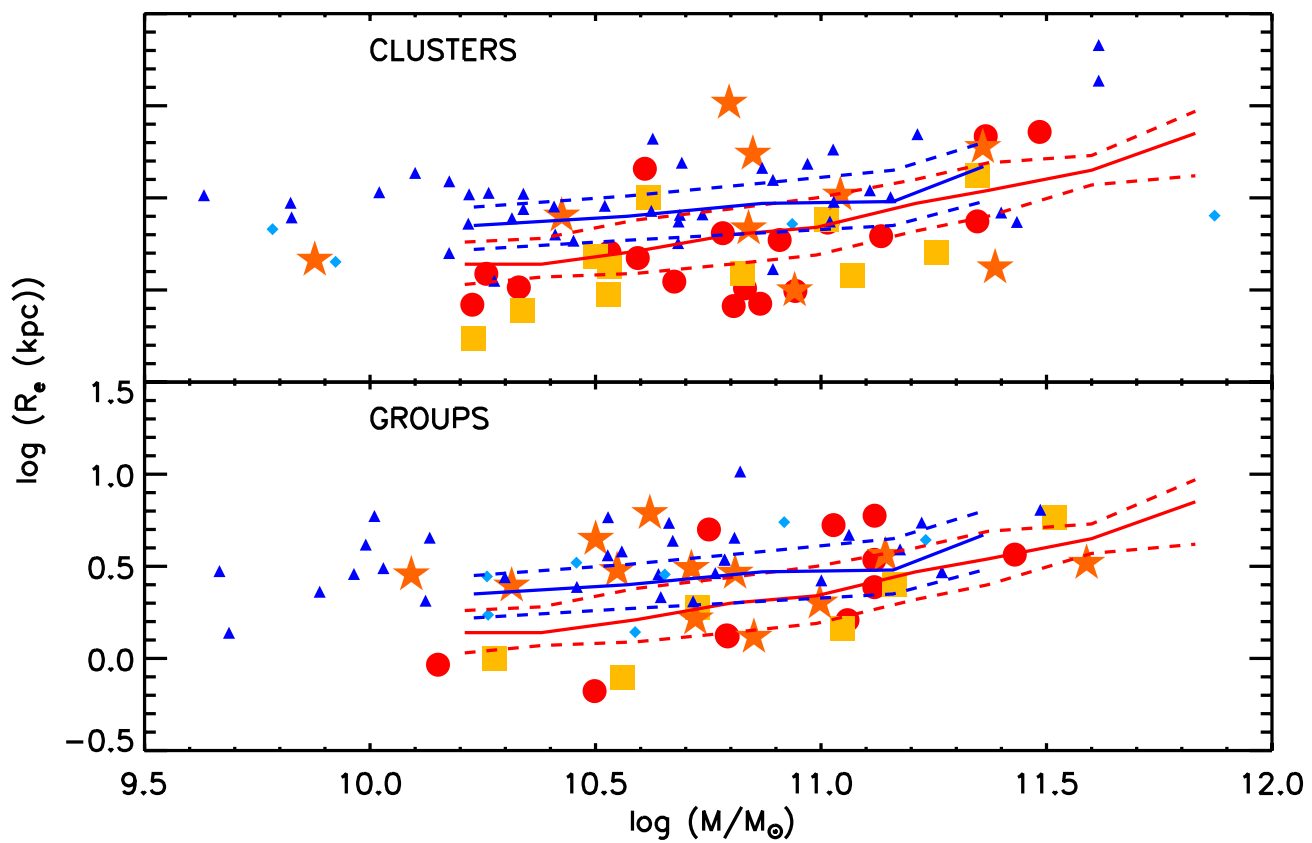

Figure 16. Mass-size relation for all galaxies in our sample (cluster and group galaxies on the top and the bottom, respectively). Our visual morphological types are shown with the same symbols as in Figure 5. The red (blue) continuous and dashed lines are the Valentinuzzi et al. (2010) ETG (disk) local relations for WINGS clusters and their $1 \sigma$ dispersion, respectively. Valentinuzzi et al. morphologies were based on a visual classification, similar to our classification of the Lynx galaxies. Cluster ETGs show a size distribution on average smaller than the Valentinuzzi et al. ETGs (Raichoor et al. 2012). Lynx Sa galaxies (orange stars), for which the fit to a Sérsic profile is dominated by their large bulges, show a size distribution larger than the Lynx ETGs. The Lynx late-type galaxies have a distribution similar to the Valentinuzzi et al. disk distribution.

(A color version of this figure is available in the online journal.)

progenitors, or have gone through transformations that enlarged their own size, e.g., by minor dry mergers (Naab et al. 2009; Shankar et al. 2011), or stellar winds and/or quasar feedback (Fan et al. 2008).
Most of our Sas do show larger sizes than ETGs, and their size distribution is in agreement with the hypothesis that (at least part of) this population would transform into early types (even if this does not demonstrate that they will). This new population 
might enlarge the ETG mass-size distribution and reproduce that observed in the local sample. Larger samples are needed to understand the relative importance of this possible transformation with respect to the accretion of larger galaxies and galaxy-galaxy transformations (major/minor-dry/wet mergers and tidal interactions).

\section{DISCUSSION AND CONCLUSIONS}

We have examined galaxy color and morphology in the clusters and the groups of the Lynx supercluster at $z \sim 1.3$. Our primary aim is to identify galaxy properties and their transformation in the cluster and group environment at redshifts where typical clusters are still being assembled.

We confirm the detection of three groups in the Lynx supercluster (Nakata et al. 2005) by XMM-Newton X-ray imaging and spectroscopic follow-up at Subaru, Keck, and Gemini telescopes. From XMM-Newton X-ray imaging, we detect Group 3 at $2.6 \sigma$ and Group 2 at $1.6 \sigma$. Group 1 shows a $0.5 \sigma$ excess, and there is $2 \sigma$ excess of the X-ray emission close to it. From the X-ray emission and scaling relations (Markevitch et al. 1998) we obtain a mass estimate for Groups 2 and 3 of $(5.7 \pm 2.6) \times$ $10^{13} M_{\odot}$ and $(5.0 \pm 1.7) \times 10^{13} M_{\odot}$, respectively, and a $95 \%$ upper limit for the mass of Group 1 at $M<4.5 \times 10^{13} M_{\odot}$. From our spectroscopic runs in the groups, we obtained redshifts for the three confirmed groups: $z=1.262 \pm 0.007$ (Group 1; from nine members), $z=1.260 \pm 0.006$ (Group 2; from seven members), $z=1.263 \pm 0.005$ (Group 3; from nine members).

These measurements confirm that these three groups are at the same redshifts as Lynx E and Lynx W, the central clusters of the Lynx supercluster (see the introduction). Group 1 is very close to Lynx W, and their galaxies are connected by an FoF algorithm. We consider the group as a separate structure, however, because its center lies at $\sim R_{200}$ from the center of the cluster and extends to $\sim 2 R_{200}$, with an area of very low density between 0.5 and $1 R_{200}$. The group might be close to merging, or in the merger process. Our present $X M M$ imaging does not permit us to detail this interaction.

We classify galaxies in the groups as early type (E, S0; ETG), late type ( $\mathrm{Sa}, \mathrm{Sb}$, and $\mathrm{Sc}$ ), and irregular and disturbed (possible mergers, tidal features), using the same visual classification as used for the two main clusters by Postman et al. (2005). We compare our visual classification to automated methods. We show that if we would have used the $\log (A)$ versus $\log (C)$ plane or the Sérsic index to separate early- from late-type galaxies, our ETG sample would have been contaminated (especially by compact BDSs, Sas) at the $30 \%$ and $40 \%$ level, respectively (see also Capak et al. 2007). More sophisticated automated classification (Huertas-Company et al. 2008, 2009, 2011) show only a $5 \%$ contamination.

From the analysis of cluster and group galaxy morphologies and colors, we find that while the CMR is already in place, this supercluster shows high fractions of red, BDSs. We classify BDSs as Sa galaxies, e.g., spirals with large bulges but with a clearly visible diffuse component that we identify as a disk but that might also be due to merger remnants and tidal features.

From analysis of the cluster and group CMR, we found lower zero points (e.g., bluer average colors) and larger intrinsic scatters in the ETG CMR for the groups, both indicating a younger galaxy population or more complex star formation histories with respect to the cluster ellipticals (e.g., Mei et al. 2006a, 2006b; 2009). We quantified this difference in age/star formation history with simple stellar population models and found the average luminosity-weighted age of ellipticals in the groups to be $\sim 0.5-0.7$ Gyr younger than cluster ellipticals. This difference in age is similar to that between the cluster core population and the peripheral ellipticals and S0s in the two clusters (Mei et al. 2009).

Our ETG sizes, ages, and star formation histories are analyzed in detail in three companion papers. In Paper II, we study cluster and group ETG formation epochs as a function of stellar mass and compare them to the field. In Paper III, we compare ETG ages and star formation histories in the two main clusters and the field. Paper IV examines the ETG Kormendy and mass-size relations. In Paper II and Paper III, we study galaxy stellar populations in detail. We find that the dependence of age on mass (older galaxies are the most massive) is more important than the dependence of age on environment (Paper II). Star formation histories in the clusters are less extended than in the field (Paper III). We also show (Paper II) the importance of understanding limitations of stellar population models when interpreting results.

We find that the early-type fraction increases with the density in the two clusters, as in other galaxy clusters at this redshift (e.g., Postman et al. 2005). However, the ETG fraction in the two Lynx clusters never rises above $\approx 50 \%$, as compared to the $\approx 80 \%$ observed in the other massive clusters at $z \approx 1$ (Postman et al. 2005; Smith et al. 2005; Desai et al. 2007). In the groups, ETG fractions never exceed $\approx 25 \%$, consistent with cluster fractions at the same densities and field fractions at the same redshift (Postman et al. 2005), and lower than what has been observed in groups (Poggianti et al. 2009; Just et al. 2010)

This lack of ETGs is compensated by the large number of red early spirals, the Sas. When we measure the bulge fractions $(\mathrm{E}+\mathrm{S} 0+\mathrm{Sa})$, we obtain fractions similar to those observed for ETGs in clusters at $z \sim 1$. Within $0.6 \times R_{200}$ (the same region used by Poggianti et al. 2010), our cluster and group ETG fractions are $56 \% \pm 9 \%$ and $23 \% \pm 9 \%$, respectively, while BDG fractions are $70 \% \pm 9 \%$ and $37 \% \pm 9 \%$, respectively.

When selecting our sample by mass, our results do not change. For galaxies with masses $M>10^{10.6} M_{\odot}$ within $\Sigma>500 \mathrm{Mpc}^{-2}$, we find an ETG fraction of $\approx 60 \% \pm 10 \%$ and $\approx 30 \% \pm 10 \%$ in the clusters and groups, respectively, and independent of the stellar population model used for the mass estimation. However, when we estimate the BDG fraction in the two clusters we obtain $\approx 90 \% \pm 10 \%$.

We compare these results to the Holden et al. (2007) ETG fractions at $z=0.83$ and lower redshifts, where the galaxy selection was made in the same way in mass and by density regions. Our results show that the ETG and bulge fractions are still high at $z \sim 1.3$. We do not see a significant evolution of the ETG and the BDG fraction either overall, or on the red sequence, from $z \sim 1.3$ to the present for galaxies with masses $M>10^{10.6} M_{\odot}$ within $\Sigma>500 \mathrm{Mpc}^{-2}$. Our results do not change if we use $M>10^{10.4} M_{\odot}$ as the mass limit.

As observed in Mei et al. (2009), a high fraction of BDS is not observed in the other clusters in the ACS IRCS. Since the fraction of ETG galaxies is changing between these two clusters and clusters at $z<1.2$, but the BDG fraction does not, as we pointed out in Mei et al. (2009), this BDS population might be thought to eventually evolve into ETGs. Since BDSs are also red and passive, they must have had, first, their star formation quenched and then subsequently experience a morphological transformation.

Recent results based on the COSMOS field (Bundy et al. 2010) show a similar trend: an increase of massive $\left(M \gtrsim 10^{11} M_{\odot}\right)$ ETGs and a decline of $M \lesssim 10^{11} M_{\odot}$ BDSs 
(in this case selected by their Sérsic index $1.25<n<2.5$ ) on the red sequence, from $z \approx 1$ to present. These authors deduced that at least $60 \%$ of the BDSs were transformed into ETGs (e.g., S0s) on the scale of 1-3 Gyr, and that these transformations might occur as a single major merger event or through multiple evolutionary stages, including disk disruption by minor mergers or accretion of cold gas in star-forming galaxies. These scales are similar to the timescales of a few Gyr derived from measurements of the S0 velocity dispersions in clusters (e.g., Moran et al. 2007).

The transformation of spirals into S0s has been largely discussed in the literature (Bekki et al. 2002; Christlein \& Zabludoff 2004; Postman et al. 2005; Poggianti et al. 2009, 2010; Wilman et al. 2009; Bekki \& Couch 2011). The most studied idea is that as they are accreted into galaxy clusters, spirals lose their disks and become ETGs. This hypothesis is difficult to reconcile with the bulge luminosity of local cluster late-type galaxies (Christlein \& Zabludoff 2004).

The BDSs that we observe, though, have luminous bulges and show an interesting mass-size relation. To test the hypothesis that ETGs might have been formed from these progenitors, we estimated the mass-size relation for our entire sample and compared it to the local relation from the SDSS. We do so by comparing, on the one hand, samples selected by Sérsic index, and on the other hand, samples selected based on visual morphology. Our results differ in the two cases. If we would have used a simple separation of early from late type, identifying ETGs as galaxies with a Sérsic index $n>2.5$, the two classes would have lied on the local mass-size relations of galaxies selected in the same way in the SDSS. Samples of galaxies selected on their Sérsic index do not show evolution in their mass-size relation.

A simple morphological classification based on one structural parameter is, however, highly contaminated by a mixing of different morphologies, with compact spirals (mainly Sas) being identified as ETGs (see Section 4). In the second case, we compare our sample to the SDSS sample, using in both cases a visual (or in case of the SDSS, trained on visual) morphological classification. As we discuss in depth in Paper IV, we find that Lynx cluster ETGs are more compact than local galaxies at a given mass (see also different results from Cooper et al. 2012 and Papovich et al. 2012). We show in this paper that spirals, however, lie on the local mass-size relation. We conclude in Paper IV that to obtain the local mass-size distribution, galaxies in clusters and groups must have either accreted larger ETGs or have gone through transformations that enlarged their own size (e.g., Fan et al. 2008; Naab et al. 2009; Shankar et al. 2011), or have formed ETGs from later type progenitors.

Most of our Sa galaxies do show larger sizes than ETGs, and their size distribution is in agreement with the hypothesis that if (at least part of) this population transformed into early types, this would enlarge the ETG size distribution and reproduce that observed in the local sample. Larger samples are needed to understand the relative importance of this transformation with respect to the accretion of larger ETGs from the field, the role of dry/wet minor mergers, wet major mergers, or strangulation of later type progenitors.

Since we do not have dynamical data for those Sa galaxies, we cannot exclude the hypothesis that the diffuse component we observe and visually identify as a disk might be tidal structures or merger remnants that will fade with time. A detailed dynamical analysis of the Sa sample would help in understanding the disk nature of the diffuse component that we observe.
Compared to field samples, Paper IV has shown that field ETGs at $z \sim 1.3$ and with similar mass limits to the present sample show a mass-size relation in agreement with the local relation. Cassata et al. (2011) have shown that, in the field, ETGs enlarge their size and increase their stellar mass by a factor of five between $z=2$ and 1. At $z \sim 1.3$, field galaxies are already on the local mass-size relation, while cluster ETGs are about twice as compact on average, indicating that their size distribution will increase (see also Strazzullo et al. 2010 for similar results at $z=1.4$, but also Papovich et al. 2012 for a different point of view, perhaps because our sample selections are different).

At the redshift of the Lynx supercluster, $\Lambda \mathrm{CDM}$ models predict that environmental effects should not yet be predominant (e.g., McGee et al. 2009). We are observing clusters before they accrete a significant number of field and group galaxies and groups before they infall into clusters. Our cluster population should be a pristine population at $z \sim 1.3$, before a significant fraction of group and field galaxies is accreted onto clusters.

Our results show a cluster population with high percentages of BDSs or bulges with a significant diffuse component (tidal structures/merger remnants; see above) that could evolve into ETGs. When we consider the fraction of this population together with the current cluster ETG population, we obtain BDG fractions that are in agreement with local BDG fractions. If these objects do transform into ETGs (e.g., S0 galaxies), the pristine ETG cluster population will not need to accrete and transform additional spirals to reach an ETG population consistent with the local population. From the observed double and triple red merger, some (we observe a few) of those red ETGs will merge to build more massive ETGs. Our results then suggest that, at these redshifts, the spiral galaxies that transform into S0s (see e.g., Postman et al. 2005; Desai et al. 2007; Poggianti et al. 2010) are mainly Sas.

The groups will eventually be accreted onto the main clusters to form a single more massive structure (with a mass typical of clusters in the local universe). Our groups show pre-processing in terms of wet similar-size mergers, the presence of small companions in two galaxies, and tidal tails and clumpy morphologies in $\sim 10 \%$ of the sample. Group BDG fractions are around $40 \%-50 \%$. If we assume pre-processing in groups in terms of transformation of BDSs into ETGs, we would need part of the disturbed morphologies to be also transformed into ETGs for the group accretions to not significantly change the total ETG fractions in clusters, as observed at lower redshift. From our sample, it appears that simple transformations of BDGs into ETGs would be in agreement with the mild evolution found for galaxies with masses $M>10^{10.6} M_{\odot}$ within $\Sigma>500 \mathrm{Mpc}^{-2}$ up to $z \sim 1.3$. Further morphological transformations that would significantly change (e.g., at $\sim 20 \%$ ) the ETG fraction should happen at lower masses and/or at lower densities.

From the analysis of the group red sequence, group ETGs also show red sequence zero point and scatter that are consistent with those of peripheral ellipticals and S0 galaxies in clusters at $z \approx 1$ (Mei et al. 2009).

\section{SUMMARY}

Our work aimed to confirm the detection of groups in the Lynx supercluster, and study galaxy morphology and 
stellar population at a time when typical clusters are being assembled.

Our main results are the following.

1. With XMM-Newton imaging and spectroscopic follow-up at Subaru, Keck, and Gemini telescopes, we confirm the detection of three groups around the two Lynx clusters, RX J0849+4452 and RX J0848+4453 at redshift $z=$ $1.262 \pm 0.007$ (Group 1), $z=1.260 \pm 0.006$ (Group 2), and $z=1.263 \pm 0.005$ (Group 3). The estimated group masses are $M<4.5 \times 10^{13} M_{\odot}($ Group 1$), 5.7 \pm 2.6 \times 10^{13} M_{\odot}$ (Group 2), and $5.0 \pm 1.7 \times 10^{13} M_{\odot}($ Group 3$)$.

2. We morphologically classify galaxies by visual inspection. Our ETG sample would have been contaminated at the $30 \%-40 \%$ level by simple automated classification methods. We observe wet mergers in both clusters and groups, and dry mergers only in clusters.

3. The Lynx supercluster shows low fractions of ETGs (when compared to other clusters at similar redshift). This lack of ETGs is compensated by high fractions of red BDSs. These objects have large bulges and a diffuse disk component that shows features similar to arms. These features might also be due merger remnants and/or tidal features. Since the fraction of ETG galaxies changes between the Lynx clusters and clusters at $z<1.2$, but the BDG fraction does not (Mei et al. 2009), this might be evidence that the BDS population will eventually evolve into ETGs (see also Bundy et al. 2010). BDSs are also red and passive: if these transformations will happen at later time, BDSs must have had, first, their star formation quenched and then subsequently experience a morphological transformation. Some of these BDSs are observed in dry mergers. If these objects do transform into ETGs (e.g., S0 galaxies), the pristine ETG cluster population will not need to accrete and transform additional spirals to reach an ETG population consistent with the local population. Our results then suggest that, at these redshifts, the spiral galaxies that transform into S0s (see, e.g., Postman et al. 2005; Desai et al. 2007; Poggianti et al. 2010) are mainly Sas.

4. In the Lynx clusters, the ETG fraction of galaxies with masses $M>10^{10.6} M_{\odot}$ within $\Sigma>500 \mathrm{Mpc}^{-2}$ do not show evolution from $z \sim 1.3$ to the present. Our results do not change if we use $M>10^{10.4} M_{\odot}$ as the mass limit. This confirms the results to the Holden et al. (2007) for the evolution of ETG fractions up to $z=0.83$

5. We studied the cluster and group CMR. The CMR is already in place in both environments. We find lower zero points (e.g., bluer average colors) and larger intrinsic scatters in the ETG CMR for the groups. Using simple stellar population models, we find that this difference corresponds to average luminosity-weighted ages of ellipticals in the groups to be $\sim 0.5-0.7$ Gyr younger than cluster ellipticals. This result is consistent with the analysis of the galaxy SEDs in Raichoor et al. (2011) and Rettura et al. (2011). However, the dependence of age on mass is more important than the dependence of age on environment (Raichoor et al. 2011).

6. We study the mass-size relation for galaxy samples selected using their Sérsic index and visual morphology. When using the Sérsic index, our results do not show an evolution of the galaxy mass-size relation, while when using visual morphology, at a given mass the ETG sizes are smaller than local cluster galaxies (see also Raichoor et al. 2012). Our result shows that galaxy samples that are morphologically selected as early based on Sérsic index are highly contaminated by a mixing of different visual morphologies and can lead to biases in results on the mass-size relation evolution. The spiral galaxy mass-size relation does not show significant evolution. At a given mass, BDSs show a large range of sizes that span both the spiral and ETG sizes. Most BDSs show large sizes, though, and their size distribution is in agreement with the hypothesis that if (at least part of) this population transformed into ETGs, it would enlarge the ETG size distribution and reproduce the ETG mass-size relation observed in the local sample.

ACS was developed under NASA contract NAS 5-32865. This research has been supported by the NASA HST grant GO10574.01-A and Spitzer program 20694. The Space Telescope Science Institute is operated by AURA Inc., under NASA contract NAS5-26555. Some of the data presented herein were obtained at the W.M. Keck Observatory, which is operated as a scientific partnership among the California Institute of Technology, the University of California, and the National Aeronautics and Space Administration. The Observatory was made possible by the generous financial support of the W.M. Keck Foundation. The authors recognize and acknowledge the very significant cultural role and reverence that the summit of Mauna Kea has always had within the indigenous Hawaiian community. We are most fortunate to have the opportunity to conduct observations from this mountain. Some data were based on observations obtained at the Gemini Observatory, which is operated by the Association of Universities for Research in Astronomy, Inc., under a cooperative agreement with the NSF on behalf of the Gemini partnership: the National Science Foundation (United States), the Science and Technology Facilities Council (United Kingdom), the National Research Council (Canada), CONICYT (Chile), the Australian Research Council (Australia), Ministério da Ciência e Tecnologia (Brazil), Ministerio de Ciencia, Tecnologìa e Innovaciòn Productiva (Argentina), and Gemini Science Program ID: GN-2006A-Q-78. R.D. gratefully acknowledges the support provided by the BASAL Center for Astrophysics and Associated Technologies (CATA) and by FONDECYT Grant No. 1100540. We thank the anonymous referee for the very constructive suggestions and Shannon Patel for useful discussions.

Facilities: HST (ACS), Spitzer (IRAC, MIPS), KPNO:2.1m (FLAMINGOS), Hale (COSMIC), Keck:I (LRIS), Gemini:North (GMOS)

\section{REFERENCES}

Abraham, R. G., Valdes, F., Yee, H. K. C., \& van den Bergh, S. 1994, ApJ, 432, 75

Arnouts, S., Moscardini, L., Vanzella, E., et al. 2002, MNRAS, 329, 355

Beers, T. C., Flynn, K., \& Gebhardt, K. 1990, AJ, 100, 32

Bekki, K., \& Couch, W. J. 2011, MNRAS, 415, 1783

Bekki, K., Couch, W. J., \& Shioya, Y. 2002, ApJ, 577, 651

Bernardi, M., Shankar, F., Hyde, J. B., et al. 2010, MNRAS, 404, 2087

Bernardi, M., Sheth, R. K., Nichol, R. C., Schneider, D. P., \& Brinkmann, J. 2005, MNRAS, 129, 61

Bertin, E., \& Arnouts, S. 1996, A\&AS, 117, 393

Bielby, R. M., Finoguenov, A., Tanaka, M., et al. 2010, A\&A, 523, A66

Blakeslee, J. P., Anderson, K. R., Meurer, G. R., Benítez, N., \& Magee, D. 2003a, in ASP Conf. Ser. 295, ADASS XII, ed. H. E. Payne, R. I. Jedrzejewski, \& R. N. Hook (San Francisco, CA: ASP), 257

Blakeslee, J. P., Franx, M., Postman, M., et al. 2003b, ApJ, 596, L143

Blakeslee, J. P., Holden, B. P., Franx, M., et al. 2006, ApJ, 644, 30 Boselli, A., \& Gavazzi, G. 2006, PASP, 118, 517

Bruzual, A. G., \& Charlot, S. 2003, MNRAS, 344, 1000 (BC03)

Bundy, K., Scarlata, C., Carollo, C. M., et al. 2010, ApJ, 719, 1969

Calzetti, D., Armus, L., Bohlin, R. C., et al. 2000, ApJ, 533, 682 
Cameron, E. 2011, PASA, 28, 128

Capak, P., Abraham, R. G., Ellis, R. S., et al. 2007, ApJS, 172, 284

Carlberg, R. G., Yee, H. K. C., \& Ellingson, E. 1997, ApJ, 478, 462

Cassata, P., Giavalisco, M., Guo, Y., et al. 2011, ApJ, 743, 96

Christlein, D., \& Zabludoff, A. I. 2004, ApJ, 616, 192

Conselice, C. J., Bershady, M. A., \& Jangren, A. 2000, ApJ, 529, 886

Cooper, M. C., Griffith, R. L., Newman, J. A., et al. 2012, MNRAS, 419, 3018

De Lucia, G., Springel, V., White, S. D. M., Croton, D., \& Kauffmann, G. 2006, MNRAS, 366, 499

Desai, V., Dalcanton, J. J., Aragn-Salamanca, A., et al. 2007, ApJ, 660, 1151

Diaferio, A., Kauffmann, G., Balogh, M. L., et al. 2001, MNRAS, 323, 999

Elston, R. 1998, Proc. SPIE, 3354, 404

Ettori, S., Tozzi, P., Borgani, S., \& Rosati, P. 2004, MNRAS, 417, 13

Evrard, A. E., Bialek, J., Busha, M., et al. 2008, ApJ, 672, 122

Faber, S. M., Willmer, C. N. A., Wolf, C., et al. 2007, ApJ, 665, 265

Fan, L., Lapi, A., De Zotti, G., \& Danese, L. 2008, ApJ, 689, L101

Fazio, G. G., Hora, J. L., Willner, S. P., et al. 1998, Proc. SPIE, 3354, 1024

Finoguenov, A., Guzzo, L., Hasinger, G., et al. 2007, ApJS, 172, 182

Gal, R. R., Lemaux, B. C., Lubin, L. M., Kocevski, D., \& Squires, G. K. 2008, ApJ, 684, 933

Gallazzi, A., Charlot, S., Brinchmann, J., \& White, S. D. M. 2006, MNRAS, 370, 1106

Gehrels, N. 1986, ApJ, 303, 336

Geller, M. J., \& Huchra, J. P. 1983, ApJS, 52, 61

George, M., Leauthaud, A., Bundy, K., et al. 2011, ApJ, 742, 125

Gobat, R., Daddi, E., Onodera, M., et al. 2011, A\&A, 526, A133

Holden, B. P., Illingworth, G. D., Franx, M., et al. 2007, ApJ, 670, 190

Holden, B. P., Stanford, S. A., Rosati, P., et al. 2001, AJ, 122, 629

Huertas-Company, M., Aguerri, J. A. L., Bernardi, M., Mei, S., \& Sánchez Almeida, J. 2011, A\&A, 525, A157

Huertas-Company, M., Rouan, D., Tasca, L., Soucail, G., \& Le Fèvre, O. 2008, A\&A, 478, 971

Huertas-Company, M., Tasca, L., Rouan, D., et al. 2009, A\&A, 497, 743

Ilbert, O., Arnouts, S., McCracken, H. J., et al. 2006, A\&A, 457, 841

Ilbert, O., Capak, P., Salvato, M., et al. 2009, ApJ, 690, 1236

Ilbert, O., Salvato, M., Le Floc'h, E., et al. 2010, ApJ, 709, 644

Jansen, F., Lumb, D., Altieri, B., et al. 2001, A\&A, 365, L1

Jee, M. J., White, R. L., Ford, H. C., et al. 2006, ApJ, 642, 720

Just, D. W., Zaritsky, D., Sand, D. J., Desai, V., \& Rudnick, G. 2010, ApJ, 711, 192

Kashikawa, N., Aoki, K., Asai, R., et al. 2002, PASJ, 54, 819

Kauffman, G., \& Charlot, S. 1998, MNRAS, 294, 705

Kells, W., Dressler, A., Sivaramakrishnan, A., et al. 1998, PASP, 110, 1487

Kirsch, M. G. F., Becker, W., Benlloch-Garcia, S., Jansen, F. A., et al. 2004, Proc. SPIE, 5165, 85

Kodama, T., \& Arimoto, N. 1997, AJ, 320, 41

Komatsu, E., Smith, K. M., Dunkley, J., et al. 2011, ApJS, 192, 18

Kuntz, K. D., \& Snowden, S. L. 2008, A\&A, 478, 575

Lubin, L. M., Gal, R. R., Lemaux, B. C., Kocevski, D. D., \& Squires, G. K. 2009, AJ, 137, 4867

Maraston, C. 2005, MNRAS, 362, 799

Markevitch, M., Forman, W. R., Sarazin, C. L., \& Vikhlinin, A. 1998, ApJ, 503, 77
Marleau, F. R., \& Simard, L. 1998, ApJ, 507, 585

McGee, S. L., Balogh, M. L., Bower, R. G., Font, A. S., \& McCarthy, I. G. 2009, MNRAS, 400, 937

Mei, S., Blakeslee, J. P., Stanford, S. A., et al. 2006a, ApJ, 639, 81

Mei, S., Holden, B. P., Blakeslee, J. P., et al. 2006b, ApJ, 644, 759

Mei, S., Holden, B. P., Blakeslee, J. P., et al. 2009, ApJ, 690, 42

Menanteau, F., Ford, H. C., Motta, V., et al. 2006, AJ, 131, 208

Moran, S. M., Ellis, R. S., Treu, T., et al. 2007, ApJ, 671, 1503

Naab, T., Johansson, P. H., \& Ostriker, J. P. 2009, ApJ, 699, L178

Nakata, F., Kodama, T., Shimasaku, K., et al. 2005, MNRAS, 357, 1357

Oke, J. B., Cohen, J. G., Carr, M., et al. 1995, PASP, 107, 375

Oke, J. B., \& Gunn, J. E. 1983, ApJ, 266, 713

Papovich, C., Bassett, R., Lotz, J. M., et al. 2012, ApJ, 750, 93

Peng, C. Y., Ho, L. C., Impey, C. D., \& Rix, H. 2002, AJ, 124, 266

Poggianti, B. M., De Lucia, G., Varela, J., et al. 2010, MNRAS, 405, 995

Poggianti, B. M., Fasano, G., Bettoni, D., et al. 2009, ApJ, 697, L137

Poggianti, B. M., von der Linden, A., De Lucia, G., et al. 2006, ApJ, 642, 188

Postman, M., Franx, M., Cross, N. J. G., et al. 2005, ApJ, 623, 721

Press, W. H., Teukolsky, S. A., Vetterling, W. T., \& Flannery, B. P. 1992, Numerical Recipes (New York: Cambridge Univ. Press)

Raichoor, A., Mei, S., Nakata, F., et al. 2011, ApJ, 732, 12

Raichoor, A., Mei, S., Stanford, S. A., et al. 2012, ApJ, 745, 130

Rettura, A., Mei, S., Stanford, S. A., et al. 2011, ApJ, 732, 94

Rettura, A., Rosati, P., Strazzullo, V., et al. 2006, A\&A, 458, 717

Rosati, P., Stanford, S. A., Eisenhardt, P. R., et al. 1999, ApJ, 118, 76

Saxton, R. D., Altieri, B., Read, A. M., et al. 2005, Proc. SPIE, 5898, 73

Shankar, F., Marulli, F., Bernardi, M., et al. 2011, arXiv:1105.6043

Schlegel, D. J., Finkbeiner, D. P., \& Davis, M. 1998, ApJ, 500, 525

Scodeggio, M. 2001, AJ, 121, 241

Shen, S., Mo, H. J., White, S. D. M., et al. 2003, MNRAS, 343, 978

Sirianni, M., Jee, M. J., Bentez, N., et al. 2005, PASP, 117, 1049

Smith, G. P., Treu, T., Ellis, R. S., Moran, S. M., \& Dressler, A. 2005, ApJ, 620, 78

Stanford, S. A., Elston, R., Eisenhardt, P. R., et al. 1997, AJ, 114, 2232

Stanford, S. A., Holden, B., Rosati, P., et al. 2001, ApJ, 552, 502

Strazzullo, V., Rosati, P., Pannella, M., et al. 2010, A\&A, 524, A17

Strüder, L., Briel, U., Dennerl, K., et al. 2001, A\&A, 365, L18

Tanaka, M., Finoguenov, A., \& Ueda, Y. 2010, ApJ, 716, L152

Tanaka, M., Kodama, T., Kajisawa, M., et al. 2007, MNRAS, 377, 1206

Tanaka, M., Lidman, C., Bower, R. G., et al. 2009, A\&A, 507, 671

Treu, T., Ellis, R. S., Kneib, J.-P., et al. 2003, ApJ, 591, 53

Turner, M. J. L., Abbey, A., Arnaud, M., et al. 2001, A\&A, 365, L27

Valentinuzzi, T., Fritz, J., Poggianti, B. M., et al. 2010, ApJ, 712, 226

van Dokkum, P. G., \& Franx, M. 2001, ApJ, 553, 90

van Dokkum, P. G., Franx, M., Fabricant, D., Illingworth, G. D., \& Kelson, D. D. 2000, ApJ, 541, 95

van Dokkum, P. G., Franx, M., Kelson, D. D., et al. 1998, ApJ, 500, 714

van Dokkum, P. G., Stanford, S. A., Holden, B. P., et al. 2001, ApJ, 552, L101

Watson, M. G., Augures, J.-L., Ballet, J., et al. 2001, A\&A, 365, L51

Wilman, D. J., Oemler, A., Jr., Mulchaey, J. S., et al. 2009, ApJ, 692, 298

Yamada, T., Koyama, Y., Nakata, F., et al. 2002, ApJ, 577, L89

York, D. G., Adelman, J., Anderson, J. E., Jr., et al. 2000, AJ, 120, 1579

Zhang, Y.-Y., Finoguenov, A., Böhringer, H., et al. 2004, A\&A, 413, 49 